\title{
LOCO: The 88-million-word language of conspiracy corpus
}

\author{
Alessandro Miani ${ }^{1}$ (D) Thomas Hills $^{2,3} \cdot$ Adrian Bangerter $^{1}$
}

Accepted: 26 August 2021 / Published online: 25 October 2021

(C) The Author(s) 2021

\begin{abstract}
The spread of online conspiracy theories represents a serious threat to society. To understand the content of conspiracies, here we present the language of conspiracy (LOCO) corpus. LOCO is an 88-million-token corpus composed of topic-matched conspiracy $(N=23,937)$ and mainstream $(N=72,806)$ documents harvested from 150 websites. Mimicking internet user behavior, documents were identified using Google by crossing a set of seed phrases with a set of websites. LOCO is hierarchically structured, meaning that each document is cross-nested within websites $(N=150)$ and topics $(N=600$, on three different resolutions). A rich set of linguistic features $(N=287)$ and metadata includes upload date, measures of social media engagement, measures of website popularity, size, and traffic, as well as political bias and factual reporting annotations. We explored LOCO's features from different perspectives showing that documents track important societal events through time (e.g., Princess Diana's death, Sandy Hook school shooting, coronavirus outbreaks), while patterns of lexical features (e.g., deception, power, dominance) overlap with those extracted from online social media communities dedicated to conspiracy theories. By computing within-subcorpus cosine similarity, we derived a subset of the most representative conspiracy documents $(N=4,227)$, which, compared to other conspiracy documents, display prototypical and exaggerated conspiratorial language and are more frequently shared on Facebook. We also show that conspiracy website users navigate to websites via more direct means than mainstream users, suggesting confirmation bias. LOCO and related datasets are freely available at https://osf.io/snpcg/.
\end{abstract}

\section{Keywords}

\section{Introduction}

Conspiracy theories (CTs) are narratives that attempt to explain significant social events as being secretly plotted by powerful and malicious elites at the expense of an unwitting population (Douglas et al., 2019; Samory \& Mitra, 2018b). Belief in CTs is widespread. In 2013, it was estimated that over $50 \%$ of the US population believed in at least one CT (Oliver \& Wood, 2014), while in 2020, in the middle of the COVID-19 pandemic, health-related misinformation attracted four times as much traffic as official health sources on social

Alessandro Miani

alessandro.miani@unine.ch

1 Institute of Work and Organizational Psychology, University of Neuchâtel, Rue Emile-Argand 11, 2000 Neuchâtel, Switzerland

2 Department of Psychology, University of Warwick, University Road, Coventry CV47AL, UK

3 The Alan Turing Institute, British Library, 96 Euston Road, London NW1 2DB, UK media (AVAAZ, 2020). The consequences associated with the circulation of such theories are not trivial, potentially leading to detrimental social action (Franks et al., 2013; Imhoff et al., 2021; Sternisko et al., 2020). Belief in CTs is linked to rejection of official information and science (Raab, Auer, et al., 2013a; Raab, Ortlieb, et al., 2013b; van der Linden, 2015), decreased intentions to adopt vaccines (Jolley \& Douglas, 2014b; Lazarus et al., 2020; Salmon et al., 2005), resistance to COVID-19 containment measures and vaccination (Biddlestone et al., 2020; Lazarus et al., 2020), and reduced protection against sexually transmitted diseases (Bogart et al., 2010). CT belief is also related to general distrust and political alienation along with endorsement of nonnormative (vs. normative) political intentions (Einstein \& Glick, 2015; Imhoff et al., 2021; Jolley \& Douglas, 2014a). Such beliefs also provide justification for engaging in everyday crime (Jolley et al., 2019; Jolley \& Paterson, 2020) and antiSemitic and Islamophobic attitudes (Golec de Zavala \& Cichocka, 2012; Swami et al., 2018). Therefore, within psychology, research has typically focused on motivational and contextual factors as well as individual differences underlying belief in CTs (Butter \& Knight, 2020; Douglas et al., 2019; Douglas \& Sutton, 2018). 
A more complete understanding of CTs requires understanding how they spread. The current focus on individual beliefs, predispositions, and biases is of limited utility in this respect, for two reasons. First, beliefs are not straightforwardly connected to CT transmission. For example, a skepticalminded individual may share a CT document within a debunking community for critical purposes (Franks et al., 2017), or a credulous individual may hesitate to share such a document in a science-oriented community for fear of being stigmatized (Lantian et al., 2018). Moreover, transmission can also be motivated strategically, independently of belief, to influence constituencies, such as when CTs or fake news is intentionally shared on social media to affect outcomes like voter behavior (Bangerter et al., 2020; Douglas et al., 2019). Second, CT beliefs do not spread per se. Rather, CTs spread as materialized forms of belief, conveyed as narratives in the form of written text (e.g., from webpages or social media posts), video (e.g., from video-sharing platforms such as YouTube), images (e.g., internet memes), or eventually audio (e.g., podcasts or the recent audio-based social media Clubhouse). Regardless of their form, CT beliefs emerge in the minds of recipients when they interact with such content. For whatever reasons CT narratives are created, they circulate, sticking in the mind of conspiracy-predisposed recipients and potentially motivating individual and collective action (Franks et al., 2013; Imhoff et al., 2021; Jolley \& Paterson, 2020). Therefore, to understand the spread of CTs and their outcomes, research should investigate the content of $\mathrm{CT}$ narratives.

On the internet, misinformation spreads faster, farther, and deeper within groups of like-minded individuals (Del Vicario et al., 2016a, b; Vosoughi et al., 2018, but see Clarke, 2007; Uscinski et al., 2018 for a critical view). The internet constitutes a system of information proliferation by which many people form opinions in regard to political parties, social issues, and health-related information (Betsch et al., 2011). In the Web 2.0 version of the internet, information is produced and consumed in a horizontal fashion, allowing anyone to create and share content, with few editorial filters (Aupers, 2012; Bessi et al., 2015a, b). CT texts may thus have the same epistemological weight for many users as mainstream texts, and compete with them for attention (Bessi et al., 2014; Eicher \& Bangerter, 2015; Hills, 2019). The perceived credibility of epistemic sources is also a function of belief in CTs (Imhoff et al., 2018). This makes epistemic authority difficult to evaluate, especially when conspiratorial narratives are promoted by political leaders (Barkun, 2017), by scholars in prestigious journals (Wakefield et al., 1998), and by Nobel Prize winners (Perez \& Montagnier, 2020).

Research on the content and circulation of CTs on the internet has focused on user-generated texts such as comments and posts gathered from social media such as Twitter (Mitra et al., 2016; Wood, 2018), Facebook (Bessi, 2016; Bessi,
Zollo, et al., 2015b; Brugnoli et al., 2019; Smith \& Graham, 2019), Reddit (Klein et al., 2018, 2019; Samory \& Mitra, 2018a, 2018b), Gab (Zannettou et al., 2018), or comment sections of news websites (Wood \& Douglas, 2013, 2015). This approach has the advantage of exploring large, ecologically valid samples of text as a complement to psychological investigations of CT beliefs. However, it is difficult to reliably extract measures of individual belief from comments embedded within the noisy and heterogeneous discussion threads of conspiracy believers and debunkers (Wood \& Douglas, 2013, 2015; but see Klein et al., 2019). Moreover, discussion threads limit the utility of extracted text because the comments and posts are brief, are contextualized in the discussion in which they are embedded, and are incapable of spreading independently from the whole thread. As a matter of fact, discussion threads are not conspiracy narratives per se. While the comments and posts they contain might instill curiosity, reinforce existing beliefs, or support conversion, they often do not constitute the actual source through which CTs are transmitted.

\section{Towards corpora of CT texts}

One valuable source of CTs for academic research aimed at understanding the content and transmission of CTs is CT websites. Although social media sites engage more traffic and are overall more popular than other websites (Facebook, Twitter, and Instagram are respectively ranked as the third, fourth, and fifth most popular websites following Google and YouTube, according to similarweb.com, accessed on 20 March 2021), websites provide more in-depth and elaborated discourse than posts and tweets on social media, which are nevertheless crucial for the spread of webpages. Conspiracy websites, specifically, are specialized sources created for the purpose of developing, collecting, and spreading CTs. These websites provide ample page space to showcase arguments that discredit official narratives and function as trustworthy epistemic sources for CT believers. Analysis of CT webpages offers a series of advantages. Webpages constitute standalone, structured texts nested within sources (i.e., websites) and as such are accompanied by paratextual (i.e., metadata) information. As standalone documents, webpages can easily be shared on social media and so can provide measures of spread. Appropriately identified webpages, therefore, would be beneficial for studying the content of CTs, and a large corpus of such CTs could provide a solid grounding for CT research.

Only a handful of studies, focused on related phenomena such as anti-vaccine movements, rumors, and fake news, have built corpora from online material (discussed below). Yet, to our knowledge, the field lacks a corpus specifically focused on online CTs. In this section, we describe published work, stressing its strengths and weaknesses.

General-purpose linguistic corpora such as the WaCky corpus (Baroni et al., 2009) and the British National Corpus 
(BNC, Aston \& Burnard, 1998), although composed of large collections of texts, do not generally allow researchers to focus on either the source type (e.g., conspiracy vs. mainstream) or a specific topic (e.g., an event that has generated a CT). It should be noted, however, that documents in WaCky were gathered from a set of 2000 seeds consisting of randomly chosen pairs of content words selected from the BNC, meaning that seeds were used as keywords to retrieve webpages. This represents a useful approach which we use here: by creating ad hoc seeds, data collection can be directed to a particular (set of) topic(s) encapsulated in the seed.

Other corpora focus on specific themes. In the field of online anti-vaccine movements, a few studies have collected webpages gathered through search engines (Fu et al., 2016; Okuhara et al., 2017; Sak et al., 2015). This approach is convenient, as it allows researchers to obtain data by mimicking how users retrieve information. However, because these corpora were collected manually, sample sizes are limited, therefore reducing generalizability. To a different degree, the CORPS corpus (Guerini et al., 2013) is composed of 3600 political speeches gathered from the web. Nonetheless, being composed of only one genre without any (matched) control group, CORPS does not enable comparisons beyond descriptive analyses.

Focusing on rumors and fake news, other studies have built corpora that include a control group that allows betweengroup comparisons (Castelo et al., 2019; Kwon et al., 2017). Kwon et al. (2017), for example, built two Twitter subcorpora from a list of rumor and non-rumor events (henceforth RumTweet). Castelo et al. (2019), on the other hand, collected material (fake news vs. mainstream) via lists of reliable and unreliable websites compiled by independent fact checkers (henceforth FNweb). This approach is useful because it reduces selection bias during data collection. However, although these two studies allow us to narrow the sampling method to obtain either two-group sources (websites) or two-group themes (rumors), they present an important limitation. In fact, to systematically study phenomena related to language (e.g., conspiracy, or fake news, or rumors) through a corpus, many forms of analysis are likely to require subcorpora matched by topic. This allows researchers to compare different versions of the same event to identify discriminating features. If not matched, the two subcorpora are treated as bags of words (as in Castelo et al., 2019, and Kwon et al., 2017), ignoring the inherent structure emerging from different themes or sources. Although some changes are expected to emerge systematically from a bag-of-words approach, there may also be important topic-specific differences. For example, differences between CT and mainstream accounts of Princess Diana's death are likely to differ in informative ways from $\mathrm{CT}$ and mainstream accounts of COVID-19. These differences can only emerge from a topic-matched corpus.
Overcoming this limitation, the PHEME dataset (Zubiaga et al., 2016) focuses on predefined events that have generated rumors, allowing researchers to compare rumor with nonrumor tweets around specific events. Yet, the only work, to our knowledge, that has focused on CTs is that of Uscinski and collaborators (2011), who gathered 100,000 published letters to the editor of The New York Times (henceforth NYT) from 1897 to 2010. After the data were collected, each document was manually coded as either referring to a conspiracy or not, of which 800 were identified as conspiracies. In addition, differently from the other works we reviewed above, the authors coded several groups of actors post hoc (e.g., left/right/foreign political actors, capitalists/communists, media, government institutions, and other).

Here, we present LOCO, ${ }^{1}$ our Language Of COnspiracy corpus that was built upon the strengths and weaknesses of the reviewed corpora.

Table 1 shows the corpora comparison, including LOCO, and summarizes each corpus' key features, including the focus (i.e., the goal, e.g., general-purpose language or fake news), the source of the material gathered (e.g., from webpages or twitter), size expressed in number of documents and tokens (i.e., non-unique words), presence of topics (e.g., events or themes) or grouping (e.g., rumors vs. non-rumors) structures, the date range of documents expressed in years, and whether the material is freely available.

\section{The language of conspiracy (LOCO) corpus}

LOCO is a multilevel topic-matched corpus composed of standalone documents $(N=96,743)$ gathered via readymade lists of conspiracy and mainstream websites (see LOCO's key feature in Table 2). LOCO has been built as a freely available text source from which researchers can extract features and/or generate predictive and classification models. Previous studies of CT textual data have extracted lexical features (Del Vicario, Vivaldo, et al., 2016b; Faasse et al., 2016; Klein et al., 2019; Mitra et al., 2016; Samory \& Mitra, 2018a; Wood \& Douglas, 2015), topic distributions (Bessi, Zollo, et al., 2015b; Klein et al., 2018; Mitra et al., 2016; Samory \& Mitra, 2018b), and narrative patterns (Samory \& Mitra, 2018b). Such analyses can be replicated and extended with LOCO due to its rich metadata.

\footnotetext{
${ }^{1}$ The acronym LOCO might suggest the idea that conspiracy theories and theorists are all crazy. Far from this position, we rather highlight the polarizing phenomenon by which, regardless of the belief position, the "others" are considered crazy. People with beliefs in CTs feel, and often are, stigmatized (Lantian et al., 2018). On the other hand, nonbelievers are also in some instances mocked as "globetards," "vaxholes," or "covidiots." The last expression is emblematic as it is used by both sides of the belief spectrum to refer to people who either believe or do not believe in COVID-19. And, of course, some conspiracy theories are true.
} 
Table 1 Key features of eight corpora relevant to conspiracy theory content

\begin{tabular}{|c|c|c|c|c|c|c|c|c|}
\hline Resource & BNC & WaCky & CORPS & FNweb & RumTweet & PHEME & NYT & LOCO \\
\hline Focus & Language & language & $\begin{array}{l}\text { Political } \\
\text { speeches }\end{array}$ & Fake news & Rumors & Rumors & Conspiracy & Conspiracy \\
\hline Obtained from & $\begin{array}{l}\text { Printed } \\
\text { material }\end{array}$ & $\begin{array}{l}\text { Web } \\
\text { pages }\end{array}$ & Web pages & $\begin{array}{l}\text { Webpages from list of } \\
\text { websites }\end{array}$ & Twitter & Twitter & Newspaper & $\begin{array}{l}\text { Webpages from list of } \\
\text { websites }\end{array}$ \\
\hline $\begin{array}{l}\text { Number of } \\
\text { documents }\end{array}$ & $4 \mathrm{~K}$ & $2.69 \mathrm{M}$ & $3.6 \mathrm{~K}$ & $14 \mathrm{~K}$ (7 K fake) & $\begin{array}{l}192 \mathrm{~K} \text { tweets } \\
\text { (61 K rumor) }\end{array}$ & $\begin{array}{l}7.5 \mathrm{~K} \text { threads } \\
\text { (35 K rumor } \\
\quad \text { tweets) }\end{array}$ & $\begin{array}{l}100 \mathrm{~K} \\
(800 \\
\quad \text { conspir- } \\
\text { acy) }\end{array}$ & $\begin{array}{l}96 \mathrm{~K} \\
\text { (24 K conspiracy) }\end{array}$ \\
\hline $\begin{array}{c}\text { Number of } \\
\text { tokens }\end{array}$ & $100 \mathrm{M}$ & $1.9 \mathrm{~B}$ & $7.9 \mathrm{M}$ & $7 \mathrm{M}^{*}$ & $2.8 \mathrm{M}^{\star}$ & $100 \mathrm{~K}^{*}$ & & $88 \mathrm{M}$ \\
\hline Topic structure & NO & $\begin{array}{l}2 \mathrm{~K} \\
\text { seeds }\end{array}$ & NO & NO & $\begin{array}{l}\text { YES } \\
111 \text { events } \\
\text { (60 rumors, } 51 \\
\text { non-rumors) }\end{array}$ & YES 9 events & YES & $\begin{array}{l}\text { YES } \\
47 \text { seeds } \\
600 \text { topics }\end{array}$ \\
\hline $\begin{array}{l}\text { Grouping } \\
\text { structure }\end{array}$ & NO & NO & NO & YES & YES & $\begin{array}{l}\text { YES } \\
\text { (matched) }\end{array}$ & YES & $\begin{array}{l}\text { YES } \\
\text { (matched) }\end{array}$ \\
\hline Year range & & & $\begin{array}{l}1917 \\
2010\end{array}$ & $\begin{array}{l}2013 \\
2018\end{array}$ & $\begin{array}{l}2006 \\
2009\end{array}$ & $\begin{array}{r}\text { Events around } \\
2014-2015\end{array}$ & $\begin{array}{l}1897 \\
2010\end{array}$ & $\begin{array}{l}1853 \\
2020\end{array}$ \\
\hline Freely available & YES & YES & YES & YES & YES & YES & NO & YES \\
\hline
\end{tabular}

Note. Resources: BNC (Aston \& Burnard, 1998); WaCky (Baroni et al., 2009); CORPS (Guerini et al., 2013); FNweb (Castelo et al., 2019); RumTweet (Kwon et al., 2017); PHEME (Zubiaga et al., 2016); NYT (Uscinski et al., 2011). *Number of tokens calculated from studies' freely available datasets

The main goal of LOCO is to shed light on the language of conspiracy. To this aim, LOCO is built on documents that revolve around CTs. Because we do not yet know what the language of conspiracy is, i.e., to what extent conspiracy language differs from non-conspiracy language, selecting documents (e.g., from webpages) based on an a priori definition of conspiracy would be difficult. At best, selecting documents based on their content would result in both a limited sample size (due to manual coding, see e.g. Fu et al., 2016; Okuhara et al., 2017; Sak et al., 2015) and limited heterogeneity (due to selection criteria based on a specific linguistic/rhetoric style). We therefore chose to categorize document selection starting from the source (i.e., websites).
Not all content from conspiracy websites will contain CTs. Intuitively, it is unlikely that all $\sim 93,000$ webpages in www. globalresearch.ca contain CTs, and some content might come from neighboring genres such as rumors, fake news, urban legends, and pseudoscience. To provide an estimate of how well conspiracy and mainstream documents reflect their true labels and how well the two sources can be distinguished from each other, we have blindly coded a subset of LOCO's documents $(60$ documents from each subcorpus, see Section SM1 in supplemental material) as being either conspiratorial or not. With an overall accuracy of .88 (Cohen's $k=.77$ ), we have correctly classified as conspiracy $85 \%$ of documents and correctly classified as

Table 2 Summary statistics of mainstream, conspiracy, and all documents in LOCO

\begin{tabular}{llll}
\hline & Mainstream & Conspiracy & Whole corpus \\
\hline No. of documents & 72,806 & 23,937 & 96,743 \\
No. of websites & 92 & 58 & 150 \\
Range of years & $1853-2020$ & $2004-2020$ & $1853-2020$ \\
& $M(S D)$ [range] & $M(S D)$ [range] & $M(S D)[$ range] \\
No. of words per document & $805.94(939)[97-9507]$ & $1236.32(1307)[100-9428]$ & $912.43(1059)[97-9507]$ \\
Total no. of words & $58,677,322$ & $29,593,678$ & $88,271,000$ \\
No. of sentences per document & $37.92(47.89)[1-1087]$ & $59.63(69.58)[1-1047]$ & $43.29(54.88)[1-1087]$ \\
Total no. of sentences & $2,760,789$ & $1,427,397$ & $4,188,186$ \\
No. of paragraphs per document & $16.56(19.30)[1-829]$ & $24.51(32.83)[1-905]$ & $18.53(23.64)[1-905]$ \\
Total no. of paragraphs & $1,205,904$ & 586,748 & $1,792,652$ \\
\hline
\end{tabular}


mainstream $92 \%$ of documents. The lower classification performance on conspiracy documents suggests that not all documents from conspiracy websites are in fact CTs, while mainstream documents are less ambiguously classified as non-conspiracy. An alternative explanation is that conspiracy texts are difficult to distinguish from mainstream texts, at least via human inspection (meaning that future algorithms might find features that help improve on human classification). These results also suggest that conspiracy and mainstream texts overlap to some extent (suggesting a continuum).

The multilevel structure of LOCO allows us to take into consideration natural hierarchical grouping of documents cross-nested within websites and topics. At the document, webpage, and website levels, LOCO's metadata ${ }^{2}$ allow researchers to create subsets of documents or to add covariates during analyses. In Table 3, we summarized the key variable types we provide with LOCO for each level. For example, each document is associated with topic labels that summarize its semantic content. These labels refer to the topics that have the highest probability (among all topics extracted from LOCO) of describing the document's content (see "Topic extraction" section). This is useful for tracking differences (e.g., in lexical features) between conspiracy and mainstream texts within a specific topic (e.g., Princess Diana's death), within a set of related topics (e.g., coronavirus outbreak in China, coronavirus outbreak in the United States), between topics (e.g., Pizzagate vs. moon landing), or within and between topics, e.g., by using a 2 (e.g., Princess Diana, coronavirus) $\times 2$ (conspiracy, mainstream) factorial design. Similar analyses can be performed using the data LOCO provides on website information about political bias, factual reporting, and website category. For most $(\sim 67 \%)$ webpages, we gathered information about their upload/creation date (see "Date" section). This allows researchers to test time-related hypotheses such as the evolution through time of topics or lexical features (e.g., coronavirus topics over time). Other crucial features of LOCO are the spread and popularity metrics associated with both websites and webpages. These metrics allow researchers to test hypotheses about social media transmission, for example, testing webpages' spread and engagement while correcting for the website's popularity. Last but not least, LOCO is provided with a set of almost 300 lexical features (e.g., psychological processes associated with words) derived from two widely used and validated text-analysis programs based on wordwithin-category counting.

\footnotetext{
${ }^{2}$ Note that we make a distinction between documents, webpages, and websites' metadata. For document, we refer to the text and its intrinsic features such as title, topic, lexical features, etc. Differently, for webpage, we refer to a set of paratextual information related to the webpage (that contains the text) such as the URL, date, spread, and the website host. Websites' metadata refer to the second level of paratextual information such as website's political bias, size, and popularity.
}

\section{Method}

\section{Seed selection}

Similar to the construction of the WaCky corpus (Baroni et al., 2009), we used seeds (i.e., keywords) to retrieve the webpages that provide the texts for LOCO. Seeds were extracted from the items of two CT-based surveys: a national poll (Jensen, 2013, Source 1, e.g., "Do you believe that Lee Harvey Oswald acted alone in killing President Kennedy, or was there some larger conspiracy at work?") and the 17-item "endorsement of conspiracy theories" from Douglas and Sutton (2011, Source 2, e.g., "The American moon landings were faked"). We extracted the seeds from these surveys for two reasons. Firstly, these surveys on CTs encompass a broad set of wellknown CTs, since they are supposed to measure specific beliefs from a wide range of people. Secondly, these surveys condense each theory within a short space, usually a sentence. These two surveys were chosen because, while they measure specific theories, they are broad in scope, and encompass a large and heterogeneous set of CTs. Items from both surveys were grouped to obtain a unique seed (e.g., "Princess Diana faked her own death so she and Dodi could retreat into isolation," "Princess Diana's death was an accident," and "One or more rogue 'cells' in the British Secret Service constructed and carried out a plot to kill Princess Diana" were merged as "Princess Diana's death").

We further broadened the pool of seeds by manually adding 20 seeds corresponding to popular (e.g., Illuminati, genetically modified organisms, Pizzagate) and current (e.g., coronavirus, Bill Gates, 5G) CTs missing from Sources 1 and 2. Note that seeds such as "chemtrails," when applied to mainstream documents, in most if not all cases return documents referring to CTs. We keep these documents in LOCO so as to have a broad mainstream pool and allow users to create subsets of texts prior to analyses (e.g., by removing mainstream documents that mention CTs, see "Mentioning "Conspiracy" and Effect of mentioning conspiracy" sections). In order to include events that might be associated with different spellings, for some seeds we used synonyms (e.g., big pharma, drug companies, and pharmaceutical industry; new world order and NWO; climate change and global warming). In Table 4, we show the full set of seeds used to retrieve documents and the final document count in LOCO by source type. Note that the seed count is larger than the number of documents. This is because a single webpage can be returned by a Google search using different keywords. For example, if a document relates to Princess Diana's death due to an Illuminati plot, then this document would be returned twice for both "Princess diana death" and "illuminati" searches.

Note that although we used seeds as keywords to retrieve webpages, we do not intend seeds to serve as proxies for document content. This is because a webpage is returned by 
Table 3 Types of variables included in LOCO

\begin{tabular}{|c|c|c|c|}
\hline Level & Variable type & Example of variable & Section \\
\hline \multirow[t]{8}{*}{ 1. Document } & \multirow[t]{3}{*}{ Raw content } & Document ID & Table 6 \\
\hline & & Title & 3.4 \\
\hline & & Text & 3.4 \\
\hline & Features & Number of words, sentences, paragraphs & 3.8 .2 \\
\hline & \multirow[t]{2}{*}{ Semantic content } & Topic & 3.6 \\
\hline & & Lexical features & 3.5 \\
\hline & \multirow[t]{2}{*}{ Conspiracy content } & Representativeness & 3.7 \\
\hline & & Mention of conspiracy & 3.8 .1 \\
\hline \multirow[t]{5}{*}{ 2. Webpage } & \multirow[t]{4}{*}{ Information } & Website host & 3.2 \\
\hline & & URL & 3.3 \\
\hline & & Date & 3.8 .3 \\
\hline & & Seeds & 3.1 \\
\hline & Spread & Facebook shares, comments, and reactions & 3.8 .4 \\
\hline \multirow[t]{4}{*}{ 3. Website } & Classification & Political orientation, factual reporting, category & 3.8 .5 \\
\hline & Size & Number of webpages & 3.8 .6 \\
\hline & Popularity & Visits, traffic, and rank & 3.8 .6 \\
\hline & Spread & Facebook shares, comments, and reactions & 3.8 .4 \\
\hline
\end{tabular}

Google if the seed is present in the webpage (but note, not necessarily in the main text) at least once. The seed presence in the webpage, however, does not necessarily indicate that the seed reflects the main topic of the document's text, because the seed can be contained in boilerplate texts or in the comments section of the webpage. Instead, we remind the user that for a more precise content of documents, we offer a more fine-grained measure of document content (extracted from the cleaned text), namely topics (see "Topic extraction" section). We include the seed variable in the LOCO dataset, believing it might be useful for answering other questions, e.g., regarding webpage indexing.

\section{Website lists}

Following previous work (Pennycook \& Rand, 2019), we gathered a list of conspiracy websites from mediabiasfactcheck (MBFC). ${ }^{3}$ Websites are labeled by MBFC as conspiracy if they publish unverifiable information related to known conspiracies such as the New World Order, Illuminati, false flags, aliens, antivaccination propaganda, etc. (for further details, see category descriptions in "Website category" section). From the whole list of 241 conspiracy websites, we selected (in December 2019) those that scored the highest on the

\footnotetext{
${ }^{3}$ https://mediabiasfactcheck.com/conspiracy/
}

conspiracy rating (i.e., "tin foil hat," $N=68^{4}$ ). This increased the chances of obtaining highly conspiratorial texts, limiting contamination by mainstream or less conspiratorial texts.

The mainstream list of websites was created (in June 2020) in a data-driven fashion by extracting the websites returned by Google for each seed. While maximizing data acquisition, this approach also mimics users' online behavior. We proceeded as follows. For each seed, we created a Google query, gathered the resulting top 40 URLs, and extracted the websites' domains. ${ }^{5}$ We repeated this operation with different IPs, mimicking the searches from the UK (London), USA (New Jersey), and Australia (Melbourne) to maximize English language domains as well as the heterogeneity of websites. This procedure returned a total of 1453 unique domains. All domain counts were aggregated, and we computed two popularity metrics per domain: (1) the number of times a domain appears overall for all seeds (absolute frequency), and (2) the number of unique seeds associated with a specific domain (relative frequency). These two metrics were chosen to obtain a large portion of pages (absolute method) and a wide coverage of seeds (relative method). The top 120 domains for each metric were visually inspected to remove potential conspiracy

\footnotetext{
${ }^{4}$ Note that the final number of conspiracy websites in LOCO is 58 . This is because during the data cleaning process for some websites we did not obtain any webpages (e.g., stormfront.org, learntherisk.org). Other websites were excluded because they were either collections of tweets and videos or were CT search engines (e.g., qanon.pub, disclose.tv, and alternativenews.com).

${ }^{5}$ E.g., telegraph.co.uk from the URL https://www.telegraph.co.uk/news/ uknews/1577644/MMR-vaccine-doesnt-cause-autism-says-study.html.
} 
Table 4 List of seeds

\begin{tabular}{|c|c|c|c|}
\hline seed source & No. of conspiracy documents & No. of & \\
\hline $5 g$ & $\mathrm{~m}$ & 702 & 1664 \\
\hline aids & 2 & 1025 & 2428 \\
\hline alien & 1,2 & 813 & 1715 \\
\hline barack obama & 1 & 496 & 1485 \\
\hline big foot & 1 & 708 & 2019 \\
\hline big pharma & 1 & 716 & 1758 \\
\hline bill gates & $\mathrm{m}$ & 717 & 1623 \\
\hline cancer & $\mathrm{m}$ & 839 & 2098 \\
\hline chemtrails & 1 & 744 & 549 \\
\hline cia cocaine & 1 & 552 & 1030 \\
\hline climate change & 1,2 & 889 & 2166 \\
\hline coronavirus & $\mathrm{m}$ & 1104 & 2588 \\
\hline covid 19 & $\mathrm{~m}$ & 1004 & 2395 \\
\hline drug companies & 1 & 1024 & 2356 \\
\hline ebola & $\mathrm{m}$ & 626 & 2140 \\
\hline elvis death & $\mathrm{m}$ & 188 & 1386 \\
\hline elvis presley & $\mathrm{m}$ & 132 & 1258 \\
\hline flat earth & $\mathrm{m}$ & 605 & 1646 \\
\hline fluoride water & 1 & 395 & 1384 \\
\hline george bush & 1 & 844 & 1737 \\
\hline george soros & $\mathrm{m}$ & 735 & 1178 \\
\hline global warming & 1,2 & 896 & 1793 \\
\hline gmo & $\mathrm{m}$ & 620 & 1924 \\
\hline illuminati & $\mathrm{m}$ & 804 & 1479 \\
\hline jfk assassination & 1,2 & 607 & 1344 \\
\hline jonestown suicide & 2 & 42 & 594 \\
\hline mh370 & $\mathrm{m}$ & 167 & 1086 \\
\hline michael jackson death & $\mathrm{m}$ & 616 & 1564 \\
\hline mind control & 1 & 949 & 2036 \\
\hline moon landing & 1,2 & 349 & 1579 \\
\hline new world order & 1 & 1036 & 2162 \\
\hline nwo & 1 & 814 & 1350 \\
\hline osama bin laden & 1 & 645 & 1415 \\
\hline paul mccartney death & 1 & 149 & 1190 \\
\hline pharmaceutical industry & 1 & 828 & 1684 \\
\hline pizzagate & $\mathrm{m}$ & 359 & 1012 \\
\hline planned parenthood & $\mathrm{m}$ & 626 & 1434 \\
\hline population control & $\mathrm{m}$ & 972 & 2295 \\
\hline princess diana death & 2 & 309 & 1338 \\
\hline reptilian & 1 & 494 & 1418 \\
\hline saddam hussein & 1 & 677 & 1623 \\
\hline sandy hook & $\mathrm{m}$ & 470 & 1500 \\
\hline september 11 attack & 1,2 & 939 & 2207 \\
\hline vaccine & 1 & 803 & 2125 \\
\hline vaccine autism & 1 & 531 & 1654 \\
\hline vaccine covid & $\mathrm{m}$ & 923 & 2031 \\
\hline zika virus & $\mathrm{m}$ & 473 & 1675 \\
\hline
\end{tabular}

Note. Sources 1, 2, and m refer to: 1 = Jensen (2013); 2 = Douglas and Sutton (2011), and m = manual 
websites (none appeared), less relevant websites such as those not related to text content (YouTube, Amazon, Instagram, Pinterest, LinkedIn, Shutterstock), websites with usergenerated content (Blogger, Facebook, Twitter), and other websites such as those related to movie reviews, private companies, and online courses. Following these exclusion criteria, a total of 19 domains were removed. Keeping all domains appearing in both metrics $(N=135)$, this list was visually inspected and subdomains were aggregated (e.g., keith.seas.harvard.edu, sitn.hms.harvard.edu, health.harvard.edu, hsph.harvard.edu aggregated to harvard.edu) while removing mistakenly extracted domains (e.g., www) and non-English domain suffixes (e.g., nationalgeographic.fr). This left us with 93 domains. ${ }^{6}$

\section{URL extraction and cleaning}

Once we had obtained the list of seeds and the two lists of websites, we proceeded with collecting the webpages' URLs through Google. Besides being the most popular search engine (ranked \# 1 worldwide according to www.similarweb. com, accessed September 2020), we used Google Search because we were interested in mimicking user behavior. Importantly, while allowing us to automate URL extraction, this procedure also uses the same search criteria for all websites, without relying on website-specific search engines that might have biased results (e.g., by using the search bar within the website).

URL scraping was performed in R ( $\mathrm{R}$ Core Team, 2019), using the curl package (Ooms, 2019). We formed Google queries by crossing each seed with each website to search for a specific seed within a specific website. For example, the Google query site:bbc.com moon landing ${ }^{7}$ returned results about moon landing from the BBC website. The UK top-level domain "google.co.uk" was chosen over "google.com" to ensure English language searches (".com" in Switzerland - where the study was conducted - automatically returns results in either German, French, or Italian). We also prompted Google to extract results in the English language by adding "hl=en" to the query. For each query, we extracted the first 60 results. Data collection occurred between May 20th and July 4th, 2020 (see workflow in SM2).

Once the URL collection was complete $\left(N_{\text {conspiracy }}=\right.$ 67,$\left.813 ; N_{\text {mainstream }}=163,488\right)$, we proceeded with removing duplicated and non-relevant URLs. This was performed by searching (with regular expressions) and removing the URLs that did not include the website searched, non-text files (pdf, pictures, videos), video and photo galleries, feeds, forums, and

\footnotetext{
${ }^{6}$ Note that this number is different from the final $N=92$ for mainstream websites. This is because after the cleaning section, the website urbandictionary.com was no longer present.

${ }^{7}$ URL: https://www.google.co.uk/search?q=site\%3Abbc.com+moon+ landing\&hl=en
}

blogs, dynamic pages (e.g., URL ending with "php," “?”), collection pages and archives of links, shops and stores, and Wikipedia lists and discussions. This procedure left us with 29,885 conspiracy and 105,461 mainstream documents.

\section{Text extraction and cleaning}

To extract the HTML files and then the useful text from our list of URLs, we tested several Python packages. These scripts, called "boilerplate stripping," remove noise text from webpages such as navigation links, header and footer sections, etc. The Python Goose package returned the best performance (see SM3) and therefore was chosen for extracting the texts. Importantly, Goose can be set to return a series of metadescriptions and tags from the raw HTML file. Therefore, along with the main body of the text, we used Goose to extract the title of the document, the language tag (further capturing non-English pages), and the date the file was uploaded on the website or created (see discussion in "Date" section).

Once all the texts were collected, we further cleaned the raw corpus using the following exclusion criteria: documents for which the HTML meta-tag language was not set as English, empty documents, exact duplicated texts, and texts shorter than 100 words. $^{8}$ In order to further remove nonEnglish documents that did not contain the language HTML tag, we removed texts in which the percentage of top 1000 English words (Fry, 2000) was below 40\% (threshold chosen after visual inspection). Finally, we also removed texts whose word count was 2.5 standard deviations above the mean of the whole corpus. This procedure left us with the final LOCO sample of 23,937 conspiracy and 72,806 mainstream documents (see Table 2 for details).

\section{Lexical feature extraction}

For each document in LOCO, we extracted measures of language use with two word-counting tools, namely LIWC (Linguistic Inquiry and Word Count, see Tausczik \& Pennebaker, 2010) and Empath (Fast et al., 2016). Both tools have been used previously to investigate the language of conspiracy on social media (Fong et al., 2021; Klein et al., 2019). These tools work on the same principle: they analyze texts, word by word, and check whether the word is included in a predefined category; if so, the category value increases. To extract LIWC categories, we used the LIWC standalone application (version 2015), while for Empath we relied on CLA (Custom List Analyzer version 1.1.1, see Kyle et al., 2015), a standalone application that, along with the batch of texts, takes

\footnotetext{
${ }^{8}$ The discrepancy with Table 2, which shows the minimum word count as 97 words in a document, is due to the fact that at this stage (document cleaning) we counted words as portions of text separated by spaces, while LOCO's final word count was performed with TAACO (see "Text statistics" section).
} 
as input an ad hoc list of dictionaries. Both tools provide standardized outputs, that is, the number of words in a given category divided by the total number of words from the text file. Note that the two tools provide different formats for their output: while LIWC returns percentages (range: 0-100), Empath returns ratios (range: $0-1$ ).

Although these tools work on the same principle, they differ in how they were built, making them somewhat complementary. First, unlike Empath, LIWC detects grammatical categories such as articles, prepositions, pronouns, etc. Second, while LIWC construction relied on human coding, Empath categories were built in a data-driven fashion from a semantic database. For instance, by seeding terms such as "facebook" and "twitter," Empath generates the category labeled "social media." The two methods by which these tools were built explain why they compute slightly different values along their categories, as shown in between-dictionary correlations (see Section SM4).

\section{Topic extraction}

For each document in LOCO, we quantify the semantic content by providing a fine-grained topical distribution. This represents a vector containing the probabilities that each of a series of topics is associated with each document. This was achieved with Latent Dirichlet Allocation, (LDA; Blei et al., 2003, see SM5 for text preprocessing). LDA is an unsupervised probabilistic machine learning model capable of identifying co-occurring word patterns and extracting the underlying topic distribution for each text document. By setting a priori the number of topics in a given corpus, LDA computes, for each document in the corpus, the probabilities for all topics of being represented in the document. Meanwhile, each word of the corpus has a probability of being part of a topic. In other words, a word $x$ has probability $\beta$ of being part of topic $k$; a topic $k$ has probability $\gamma$ of being part of document $n$. The sum of all the word probabilities within one topic is 1 , and the sum of all the topic probabilities within one document is 1 .

In LDA, the "right" number of topics is determined by the goal of the task more than the data itself (Nguyen et al., 2020, but see also clustering algorithms in general; von Luxburg et al., 2012). LDA topics can be thought as the resolution of a microscope (Barron et al., 2018; Nguyen et al., 2020): if a fine-grained resolution is required, then a large number of topics is better; if the number of topics is small, these topics become more general (Allen \& Murdock, 2020). Here, topic extraction was performed with the topicmodels $\mathrm{R}$ package (Grün \& Hornik, 2011), using Gibbs sampling. We left the other LDA parameters set as default, while setting the same seed for reproducibility for all topic extractions. We performed topic extraction with three different levels of resolution, setting $k$ at 100, 200, and 300 topics. As a consequence, summing all $k$ topics, we obtained 600 topics (see
Section SM6 for a thorough description of topics and Section SM7 for topic comparison between different $k$ s). In Section SM7.1 of the supplemental material, we have suggested a way to assess topic specificity based on the position of a theme's keyword (e.g., "Diana" for Princess Diana) within the beta weight-ordered topic's terms, and the correlation with lexical features. If the theme is event-based (e.g., disappearance of Malaysia Airlines Flight 370 [MH370], 8 March 2014), we also suggest visually inspecting the gamma values plotted over time.

As a proxy for document topic, for each of the three sets of $k$ topics, we extracted the topic that had the highest probability of representing the document, i.e., the highest gamma value within all topics within $k$, and included it in the LOCO dataset (see dataset description in "Data availability" section). This means that each document is associated with three topic labels, one for each $k$. We chose this option so as to offer LOCO users a way to perform analyses on a specific topic resolution. Note that we did not provide labels for document topics. Instead, we provide the top 15 words for each topic that, taken together, summarize the topic's content (Nguyen et al., 2020, and see also beta weight distributions by $k$ in Section SM6.1).

We provide with LOCO the matrix containing all gamma values for each document and topic pairs (see "Data availability" section). This results in a matrix with a dimensionality of 96,743 documents $\times 600$ topics. This is useful for obtaining a fine-grained topic description for each document. For example, if a document $n$ has the topic with the highest $y=.90$, then this topic has $90 \%$ probability of representing document $n$, while the remaining $10 \%$ is distributed among all other topics. Similarly, if the highest $y=.10$, all the other topics, by exclusion, occupy the remaining $90 \%$ of probabilities. While in the first case we can say that document $n$ is well represented by a topic $k$ (where gamma is maximum), in the second case, the low gamma value shows that the document $n$ is not well represented by a topic $k$. LOCO contains all $\gamma$ values, allowing the user to select their own threshold when selecting documents based on topic.

\section{Data associated with LOCO's topics}

In order to facilitate topic exploration prior to data analysis, we attach additional files to LOCO that offer an in-depth description of topic content. The first one is a matrix that contains all gamma values for each topic for each document (topic gamma.json). Because there are three sets of $k$ topics $(100,200$, and 300), we have named each topic adding the $k$ resolution as prefix. For example, the fifth topic of k200 is labeled "k200 5," while the 134th topic of k300 is labeled "k300_134." Note that, because we merged the three sets of $k$ s into a unique dataset, the sum of topic probabilities for each document is now 3 ( 1 for each $k$ set of topics). The second file (topic_description.json, see also description in SM6) includes 
descriptions for each of the 600 topics. Descriptions include the top 15 terms ordered by beta weight, the number of documents in which the topic has the highest gamma, the highest correlation with other topics and highest correlation with lexical features (both LIWC and Empath). We also provide a series of plots (in the file "topic_by_time.pdf," see description in SM6), one for each topic, that track the evolution through time (from 1995 to 2020, see e.g. Fig. 2) of the gamma values. Each plot also includes the topic name and the list of the top 15 terms, ordered by beta values. We believe that these plots, along with the description of each topic (and the actual matrix with gamma values), will help researchers not only in exploring topic associations and lexical features, but also in visually inspecting topics prior to data analysis.

\section{Representative conspiracy theories}

Because one might be interested in what a prototypical conspiratorial language is, we aimed at extracting a set of the most representative CT documents on the basis of the most frequently occurring words within the conspiracy subcorpus. We believe that a set of representative documents may allow researchers to make inferences about CTs more generally. As such, a representative document should share more words with the conspiracy subcorpus compared to a less representative document. Recurrent word patterns such as "they are trying to KILL US!" (from document C01b90) or "know the truth" (document C073a0) might in fact be highly shared across conspiracy documents; hence they would be represented to a larger extent in the conspiracy universe.

Following this reasoning, we extracted the documents that were most similar to the entire conspiracy subcorpus. As a measure of representativeness, we computed the cosine similarity (CS) between words of each document against all words in the conspiracy subcorpus (for a similar procedure, see e.g. de Vries et al., 2018). Text preprocessing was the same as we used to extract LDA topics (see SM5). Documents' CS was computed using the textstat simil function from the $\mathrm{R}$ package quanteda. Values range from 0 to 1 , indicating either no overlap (0) or a perfect overlap (1) of terms. This returned a vector for each conspiracy document that indicated the similarity between it and all other conspiracy documents. We averaged this vector to obtain a single value for each document. We finally labeled as "conspiracy representative" the documents whose CS value was higher than one standard deviation above the mean. This resulted in a subset of 4,227 documents, that is, $17.66 \%$ of the conspiracy subcorpus. In Section SM8 of the supplemental material, we report the top five documents with the highest and lowest cosine similarity.

\section{Metadata}

\section{Mentioning "conspiracy"}

We marked documents that mentioned conspiracy in the text. This was done by searching, via regular expressions, and counting the occurrences of the word "conspir*." This measure helps keep track of mainstream documents that mention conspiracy which may contaminate mainstream language with details about the corresponding conspiracy (e.g., Pizzagate or Illuminati, themes that rarely appear outside the context of CTs). Therefore, instead of removing these documents, as they represent a special case of mainstream media whose focus is on CTs, we left them in LOCO and annotated the number of instances of the word "conspir*." subcorpus, a total of 3520 documents mentioned conspiracies at least once, while in the mainstream subcorpus there were 5031 documents. On average, conspiracy documents show more instances of "conspir*" than mainstream documents (conspiracy: $M=0.351, S D=1.548$, range: $0-75$; mainstream: $M=0.211, S D=1.735$, range: $0-182, t_{(45246)}=$ $11.773, p<.001, d=0.09$ ). However, when the instances were normalized per word count (i.e., divided by numbers of words in text), there were no differences, $t_{(49107)}=.993, p=$ $.321, d=0.01$.

\section{Text statistics}

For each document, we calculated the number of words, sentences, and paragraphs using the Tool for the Automatic Analysis of Cohesion, TAACO (Crossley et al., 2016, 2019), a freely available standalone application that allows batch processing of text files. Although LIWC also provides measures of word count, which correlates highly with TAACO word count, $r=.9996$, we relied on TAACO measures for two reasons. First, based on the Python Natural Language Toolkit (Bird et al., 2009), TAACO extracts the part of speech for each word, from which it derives a text word count as well as the number of sentences and paragraphs. This, we believe, is a more sophisticated way than merely counting instances of characters separated by spaces. Secondly, because the wordper-sentence measures of LIWC and TAACO correlate poorly, $r=.59$, we visually inspected documents with the highest discrepancy between the two tools. We discovered that LIWC performs poorly when full stop periods are missing from sentences, whereas TAACO considers the new line as a valid

\footnotetext{
${ }^{9}$ The word "conspir*" was chosen to be able to retrieve all conspiracy-related words (conspiracies, conspiracist, conspiracy, conspiration, conspirator, conspiratorial, conspiratorially, conspiratress, conspire, conspirer, and conspiring) but not others (e.g., conspicuous). This was checked on both American and British Oxford English dictionaries.

${ }^{10}$ From this count measure, a Boolean measure of "mentioning conspiracy" can easily be derived by simply stating "TRUE if mentions $>0$."
} 
sentence-separator marker. Therefore, in LOCO, we keep both LIWC and TAACO word counts, but for consistency with paragraph and sentence counts, we report here (see Table 2) only the TAACO word count.

\section{Date}

Information about document date was obtained primarily from the Goose package, which extracts the upload date directly from the raw HTML document. When date was not available (i.e., Goose returned an empty cell), we extracted the upload date with regular expressions from the URL of the document (e.g., "http://[...]/2018/01/23/[...].html" was coded as 23 January 2018). In LOCO, date data are provided for 63,868 documents (67\% of the entire corpus; $56.67 \%$ conspiracy and $69.09 \%$ mainstream), see distribution of documents by date in Fig. 1.

It must be noted that date values reflect either the upload date or the authoring date. Both types of information would be informative for different purposes: texts that were authored on the same date are based on a similar level of available information/evidence; texts that were published on the same date compete for audience attention. ${ }^{11}$ While dates before the internet era (e.g., 1853) refer unambiguously to the authoring date, this is less clear for more recent documents. We believe that this information might be nevertheless useful, and therefore we provide all dates available in LOCO. We warn researchers to be aware of date ambiguity before testing any time-related hypothesis. Researchers can either set a threshold for documents' dates to keep (e.g., after the internet became widespread or another arbitrary cutoff) or develop a method to disentangle the two. However, although documents' dates may refer to either authoring or upload date, we show in Fig. 2 that documents' dates are nevertheless linked to the social events discussed in documents.

Lastly, date range differs between mainstream and conspiracy subcorpora, see Table 2. We do not know the reason for this difference, considering that our Google search was independent from documents' upload date. One possible explanation is that conspiracy websites, being less popular (see Table 5), are also developed with less standardized protocols (see e.g., www.w3c.org). This might have resulted in a less methodical use of HTML meta-tags and therefore the lack of date in some documents. This might also explain the higher percentage of missing dates in conspiracy documents $(56$. $67 \%$ ). If this is the case, some documents predating 2004 (i.e., the oldest conspiracy document in LOCO) might be in this corpus yet lacking the date. Alternatively (or complementarily), conspiracy websites might be younger, overall, than mainstream websites. For example, the infowars.com domain was registered on 1999-03-07 (data obtained from https://

\footnotetext{
${ }^{11}$ We thank the anonymous reviewers for this suggestion.
}

who.is), 911truth.org on 2003-01-14, ahtribune.com (less popular in terms of monthly visits among LOCO's conspiracy websites) on 2015-08-23, and worldaffairsbrief. com (most popular) on 2004-04-06. In contrast, scientificamerican.com was created on 1997-05-02, sciencemag.org on 1996-04-28, cnn.com on 1993-09-22, and bcc.com on 1989-07-15. Although not tested systematically, those few observations suggest that, overall, conspiracy websites in LOCO might be younger than mainstream ones, therefore explaining the different date ranges.

\section{Facebook shares}

For each webpage, we obtained information about spread from the web tool sharedcount.com (SC). Via an application programming interface, SC retrieves from Facebook ${ }^{12}$ the number of shares, comments, and reactions for each webpage URL. According to the website, SC reports "all time statistics," which means that values refer to the overall shares since the creation of the URL tracked. All data from SC were collected in September 2020.

Besides single URL shares, we also computed an estimation of the total number of shares from the observed data we collected for each website. To this end, we computed the sum of all webpage Facebook shares for each website and divided them by the proportion of sampled LOCO webpage for each website. For instance, in LOCO, there are 967 documents extracted from the website www.infowars.com. Infowars has 15,500 webpages indexed on Google (see "Website metrics" section), which means that LOCO contains $6.24 \%$ of all Infowars webpages. The aggregated total Facebook shares of all 967 Infowars documents in LOCO is 89,639. By dividing the total shares $(89,639)$ for the proportion of LOCO documents (0.0624), we obtain an estimation of total website shares, which in this case is $1,436,820$ times, a rough estimation of the grand total of shares of all Infowars webpages. Once this measure was computed for all websites, we then tested the correlations of this measure with other spread measures. The estimated Facebook shares correlates with website global rank $(r=-.81)$ and with website monthly visits ( $r=.81$, see SM9 for more details).

\section{Website category}

We relied on MBFC for obtaining metrics of political side and factual reporting for each website. MBFC contains manual annotations and bias analyses for over 2,000-mostly news-websites. According to the MBFC method, ${ }^{13}$ each website's bias is evaluated on four criteria, including biased

\footnotetext{
12 see: https://developers.facebook.com/tools/debug/)

13 https://mediabiasfactcheck.com/methodology/
} 


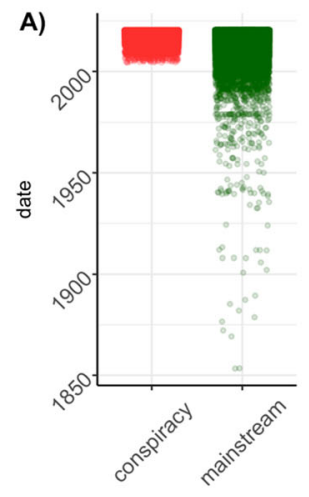

B)

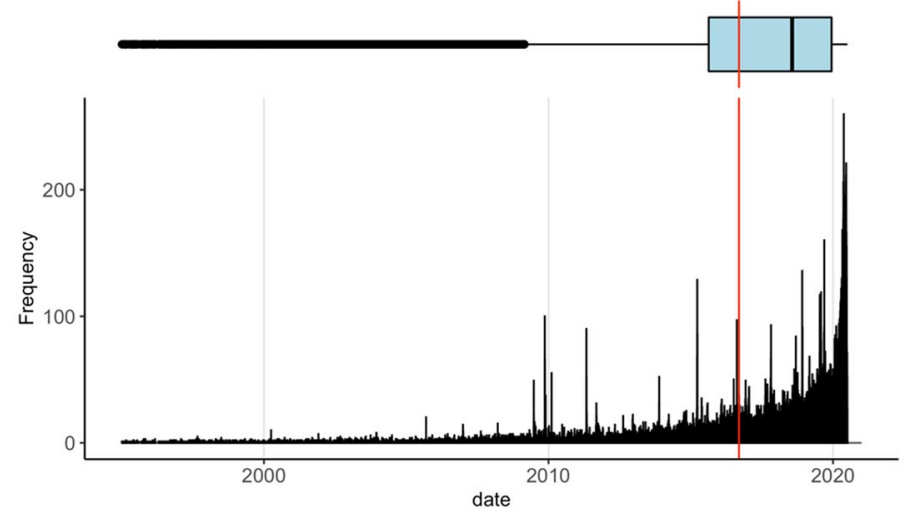

Fig. 1 Distribution of documents in LOCO by date. Distribution for a each subcorpora (red: conspiracy; green: mainstream) and $\mathbf{b}$ all documents from 1995 to the time of data collection (the red vertical line represents the mean, the boxplot on top displays the median and the interquartile ranges)

wording headlines (e.g., the source uses loaded words to convey emotion to sway the reader), factual sourcing (e.g., the source reports factually and backs up claims with wellsourced evidence), story choices (e.g., the source reports news from both sides), and political affiliation (e.g., the source endorses a particular political ideology). Factual reporting is based on the factual sourcing used for assessing bias. For each website, a minimum of 10 headlines and 5 news stories are assessed by MBFC experts. Low and very low factual reporting sources are those that need to be fact-checked for intentional fake news, conspiracy, and propaganda. Although MBFC states that their methodology has been not tested scientifically, they nevertheless adhere to the International FactChecking Network fact-checkers' Code of Principles ${ }^{14}$ and strive for transparency. Furthermore, MBFC annotations have been used by other researchers to study fake news and conspiracy websites (Baly et al., 2018; Cinelli et al., 2021; Pennycook \& Rand, 2019; Risius et al., 2019).

For each of the LOCO websites that was reviewed in MBFC, we extracted measures of political orientation (left, left center, least biased, right center, and right), factual reporting (from "very low" to "very high"), pseudoscience level (provided by MBFC only for conspiracy websites), and whether the website was labeled as pro-science (i.e., relying on legitimate science or evidence based on credible scientific sourcing). Note that pro-science websites do not have political orientation labels. Data from MBFC were collected in July 2020.

\section{Website metrics}

We have extracted a series of website metrics that, overall, offer an idea of popularity, engagement, and size for each website. From the web tool similarweb.com ${ }^{15}$ (SW), we collected data about monthly total visits, global rank, and

\footnotetext{
$\overline{14} \mathrm{https}: / /$ www.poynter.org/ifcn/

15 https://www.similarweb.com/corp/ourdata/
}

category. We also collected information about the type of incoming traffic. Expressed in percentage, these metrics partition each website's incoming traffic into direct (when a user reaches the website directly by typing the URL on the web browser or recalling it from bookmarks), from a search engine (when a website is reached through a search engine, e.g., Google), and from social media (when a website is reached through social media, e.g., a post on Facebook or Twitter). Other types of incoming traffic offered by SW, which we did not collect, are referrals, mail, and display, which overall account for about $7 \%(\mathrm{SD}=6.38)$ of remaining incoming traffic in our dataset (computed by summing direct, search engine, and social media traffic and subtracting it from 100).

SW was chosen over Alexa.com (a web tool that provides similar services), mainly because SW updates its statistics every month, whereas Alexa provides daily updates. While the latter appears to be more fine-grained, it nevertheless poses some limitations in terms of data collection (which manually spans several days) due to daily statistical fluctuations. In addition, SW offers a wide range of free features, otherwise accessible in Alexa upon a monthly subscription, and, importantly, the SW database is composed of $\sim 50$ million websites (vs. $\sim 30$ million websites in Alexa). These data were collected in July 2020.

In addition, in order to obtain an estimation of the website size, we extracted the total number of webpages per website indexed by Google. This was done by querying Google with "site:" followed by the website. ${ }^{16}$ This data was collected in March 2021.

\section{Data availability}

LOCO's data is freely available at https://osf.io/snpcg and includes:

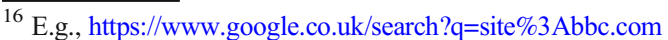


k300_50

bin, Iaden, al, osama, qaeda

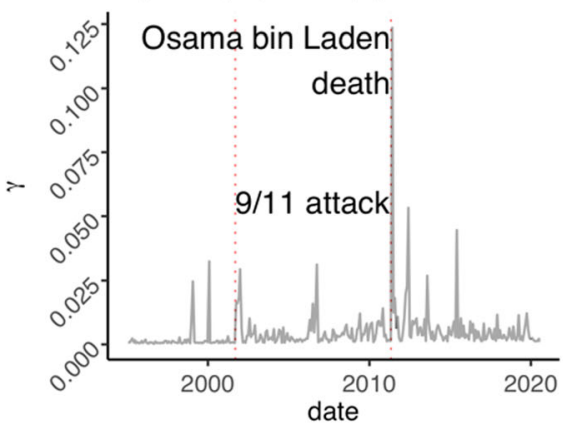

k300_134

iraq, saddam, iraqi, hussein, al

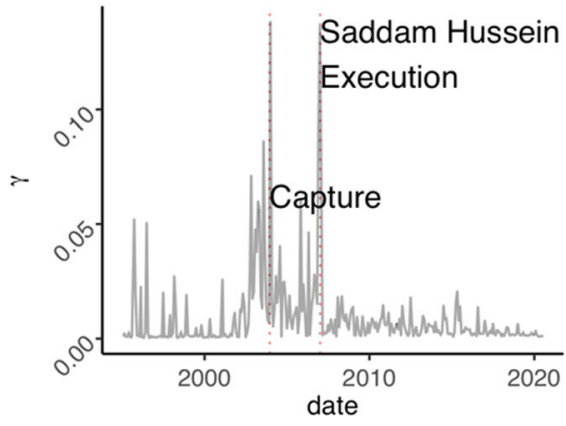

k300_146

bush, presid, georg, his, he

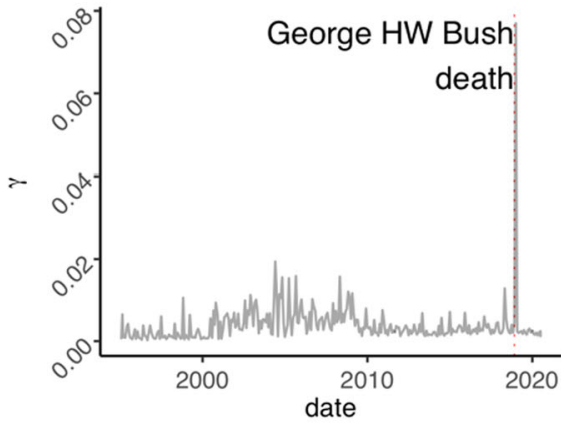

k300_200

coronavirus, covid, pandem, virus, sar

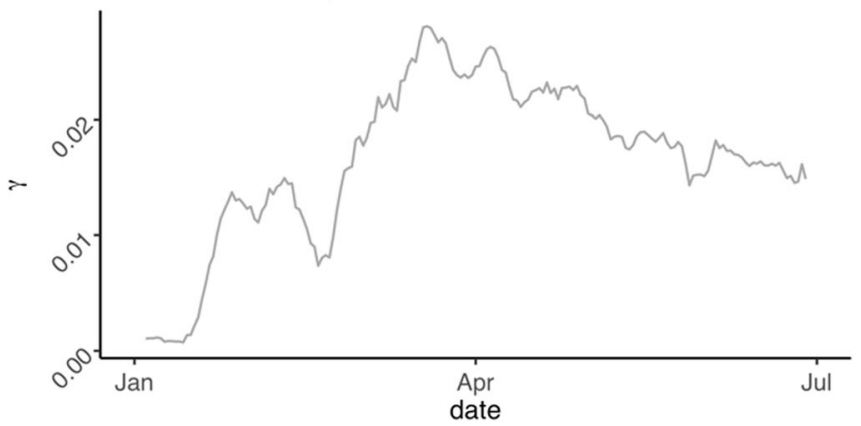

Fig. 2 LDA topic gamma values over time. The red dotted vertical lines represent the occurrences of significant events associated with the topic. In the 9/11 topic, each vertical line represents September 11th in each

k300_192

k300_72 k300_290

gun, shoot, school, hook, sandi

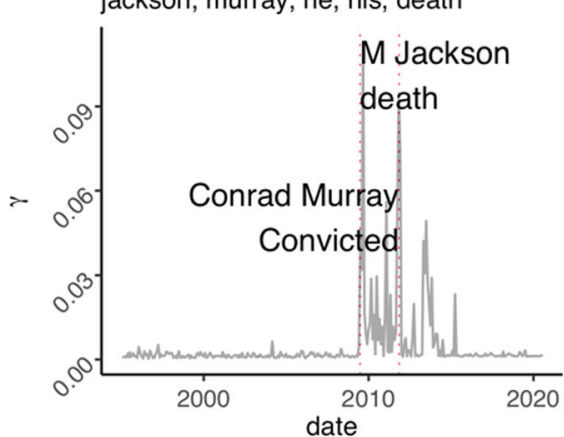

abus, epstein, sex, child, he
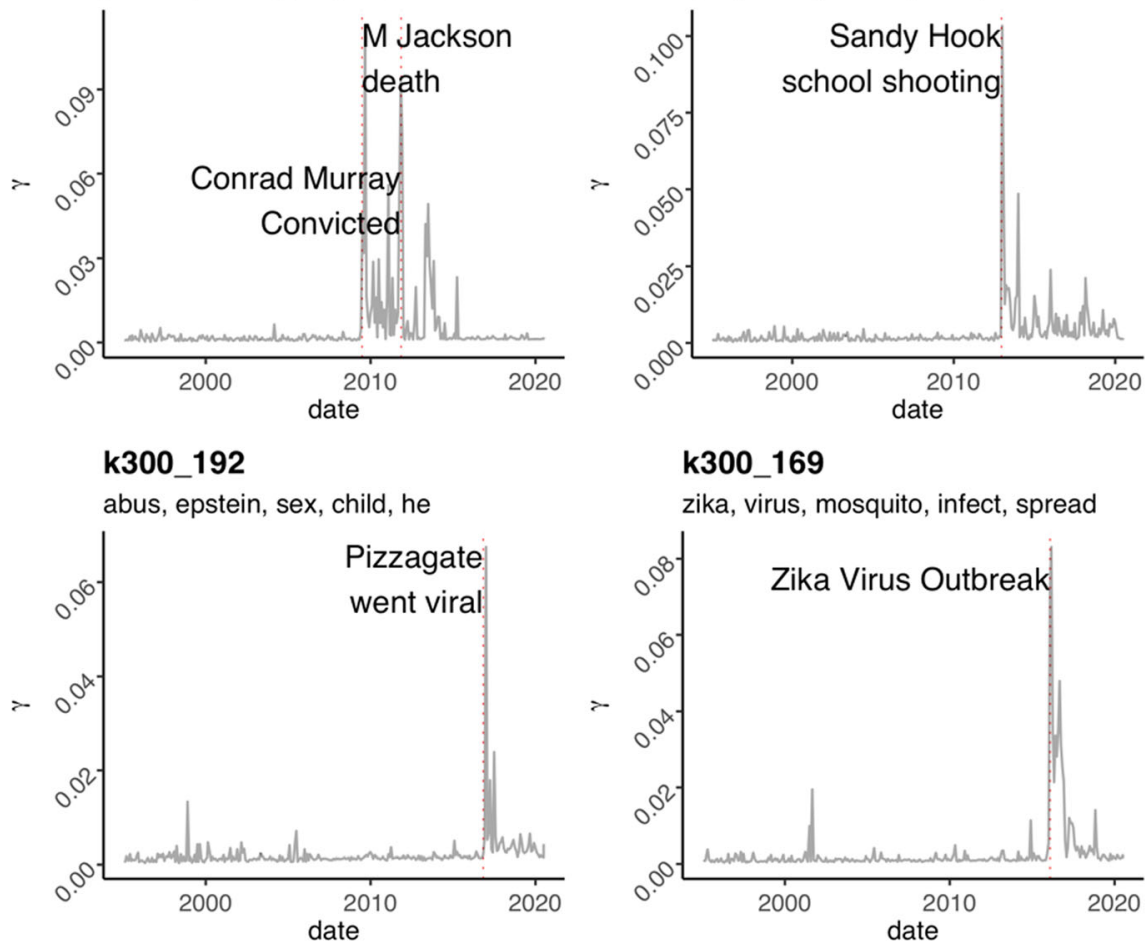

k300_169

zika, virus, mosquito, infect, spread

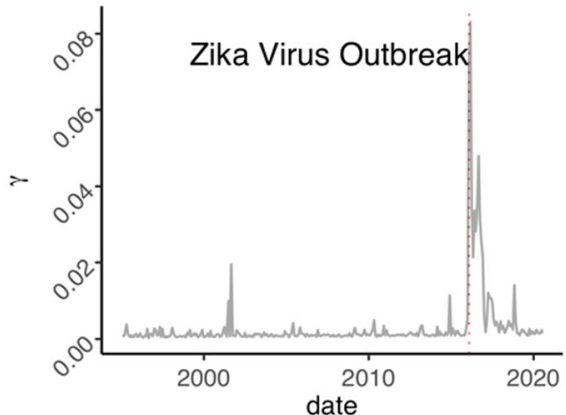

attack, tower, center, trade, world

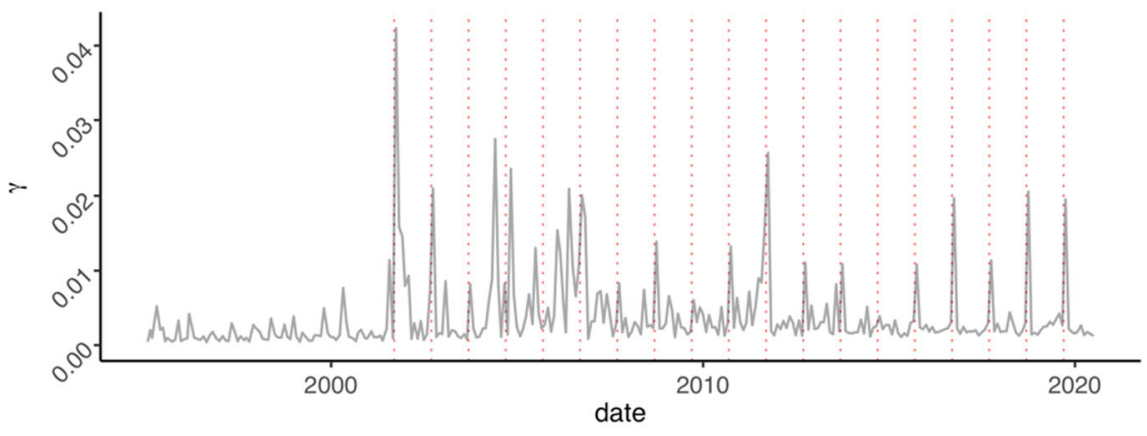

k300_288

coronavirus, covid, pandem, peopl, lockdown

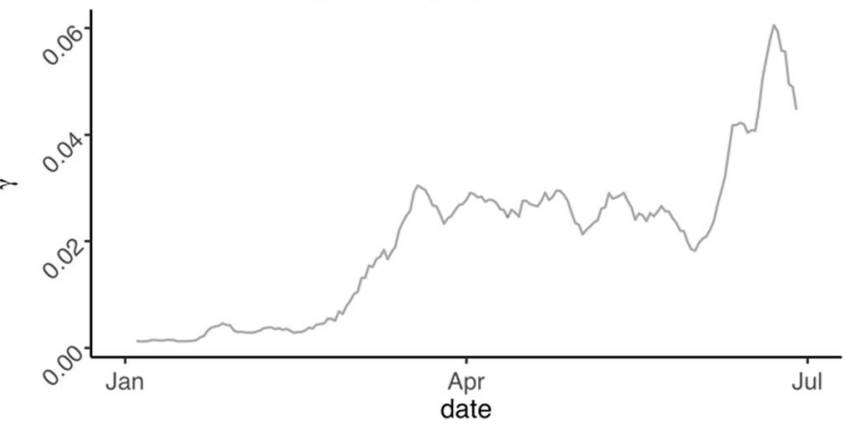

year, starting from 2001. Coronavirus topics (bottom) are distributed over the year 2020 (from January to July, when LOCO data collection ended). 
Table 5 Differences between conspiracy and mainstream website metrics

\begin{tabular}{|c|c|c|c|c|c|c|c|c|c|c|c|c|}
\hline & \multicolumn{3}{|l|}{ Mainstream } & \multicolumn{3}{|l|}{ Conspiracy } & \multicolumn{3}{|c|}{$t$-test statistics (raw) } & \multicolumn{3}{|c|}{$t$-test statistics $(\log )$} \\
\hline & $M$ & $(S D)$ & $N$ & $M$ & $(S D)$ & $N$ & $t$ & $p$ & $d$ & $t$ & $p$ & $d$ \\
\hline Total monthly visits & $102,285,513$ & $(191,306,614)$ & 92 & 965,242 & $(2,115,315)$ & 28 & 5.08 & $* * *$ & 1.10 & 14.00 & $* * *$ & 3.02 \\
\hline Global rank & 7313 & $(21,765)$ & 89 & 211,904 & $(168,890)$ & 28 & -6.39 & $* * *$ & 1.39 & -17.11 & $* * *$ & 3.71 \\
\hline Website size & $6,844,908$ & $(16,049,205)$ & 92 & 6224 & $(12,918)$ & 58 & 4.09 & $* * *$ & 0.69 & 18.43 & $* * *$ & 3.09 \\
\hline FB projected shares & $3,213,458,353$ & $(9,348,074,961)$ & 92 & $27,11,190$ & $(14,540,274)$ & 58 & 3.29 & $* *$ & 0.55 & 12.78 & $* * *$ & 2.14 \\
\hline Traffic, direct ${ }^{\dagger}$ & 28.95 & $(13.45)$ & 92 & 57.55 & $(21.57)$ & 28 & -6.63 & $* * *$ & 1.43 & & & \\
\hline Traffic, search ${ }^{\dagger}$ & 56.83 & (17.49) & 92 & 13.82 & (10.44) & 28 & 16.00 & $* * *$ & 3.45 & & & \\
\hline Traffic, social $^{\dagger}$ & 8.08 & $(5.58)$ & 92 & 18.4 & (19.28) & 28 & -2.8 & $* *$ & 0.60 & & & \\
\hline
\end{tabular}

Note. Differences tested with Welch's unequal variances $t$-test. Log transformation was applied to highly skewed variables after having added a constant 1 to avoid -Infinite values when the raw score was zero. ${ }^{\dagger}$ Values expressed as percentages and not log-transformed. $d$ : Cohen's $d$. FB: Facebook. Website size is expressed in number of webpages

1. LOCO.json (587.6 MB): a JSON (JavaScript Object Notation) file containing the LOCO corpus itself. 96,746 rows (documents) $\times 20$ columns (see Table 6 )

2. website_metadata.json $(55.3 \mathrm{~KB})$ : a JSON file containing websites' metadata. 150 rows (websites) $\times 18$ columns (see Table 7)

3. LOCO_LFs.json (573.1 MB): a JSON file containing the full set of lexical features. 96,746 rows (documents) $\times 288$ columns $\left(N_{\text {Empath }}=194 ; N_{\text {LIWC }}=93\right)$

4. topic_gamma.json (963.7 MB): a JSON file containing topics' gamma values. 96,746 rows (documents) $\times 600$ columns (topics)

5. topic_by_time.pdf (169.6 MB): a PDF file containing plots of topics' gamma values over time (from 1995 to 2020). It contains 600 pages.

6. topic_description.json (188.2 KB): a JSON file containing detailed descriptions of topics. 600 rows (topics) $\times 12$ columns (see SM6)

\section{Exploring LOCO's features}

In this section, we explore LOCO's features and provide examples on how to handle LOCO's variables and subset corpus. Some of these analyses are descriptive in nature and offer a way to visually explore to what extent LOCO's data relate to the external world, such as visualizing the evolution of LDA topics through time (see "Topic analyses" section) or exploring to what extent the language used in LOCO's documents overlaps with the language used in social media (see "Towards corpora of CT texts" section). Other analyses are more explorative, such as testing whether mentioning conspiracy in mainstream documents affects lexical features (see "Effect of mentioning conspiracy" section) or whether conspiracy-representative documents are in fact different from other conspiracy documents in terms of lexical features and spread (i.e., Facebook shares, see "Properties of representative conspiracy documents" section). Lastly, we also explore to what extent LOCO's higher-level metadata might provide insights into psychological processes by analyzing the behavior of websites' users (see "Website incoming traffic" section). Overall, these analyses not only suggest how to use LOCO, but also offer insights on the language of conspiracy and the psychology of conspiracy websites users.

\section{Topic analyses}

Each document in LOCO is associated with a vector that encapsulates and quantifies the semantic content, namely the LDA topics. While in the main dataset (LOCO.json) we provide for each document only the label of the most prevalent topic (one for each level of topic resolution, that is $k=100, k$ $=200$, and $k=300)$, in a separate dataset (topic_gamma.json) each document is associated with the gamma values for all 600 LDA topics extracted. In this section, we explore how LDA topics reflects real-world events by visually inspecting how these LDA topics develop through time for documents whose date was recorded. This reasoning is supported by the fact that, because texts are capable of showing cultural patterns (Lansdall-Welfare et al., 2017; Li et al., 2019, 2020; Michel et al., 2011), a significant social event should be reflected in the texts' topic time series. To explore this possibility, we selected a set of topics that are associated with a specific event (instead of non-eventspecific topics such as AIDS or Illuminati) such as the death of societally significant people: Osama bin Laden (2011-0502, topic k300_50), Michael Jackson (2009-06-25, k300_18), George H.W. Bush (2018-11-30, k300_146), and Saddam Hussein (2006-12-30, k300_134); outbreaks of pandemics such as Zika virus (2016-02-01, k300_169) and coronavirus 
Table 6 LOCO dataset variables description

\begin{tabular}{|c|c|}
\hline $\begin{array}{l}\text { Variable name (\% empty/missing } \\
\text { values, if any) }\end{array}$ & Variable description \\
\hline doc_id & $\begin{array}{l}\text { Six-character hexadecimal sequence of document unique identification number. The first character stores the source: C } \\
\text { stands for conspiracy (e.g., C0004d) and M stands for mainstream (e.g., M095eb) }\end{array}$ \\
\hline URL & URL associated with the document \\
\hline Website & The website from which the document was extracted \\
\hline seeds $(2.26 \%)$ & The seeds we used to gather documents. The page was returned by all the keywords listed in this variable $(N=47)$ \\
\hline date $(33.98 \%)$ & The date the webpage was uploaded or uploaded (format: YYYY-MM-DD) \\
\hline subcorpus & Either conspiracy or mainstream $\left(N_{\text {conspiracy }}=23,937 ; N_{\text {mainstream }}=72,806\right)$. \\
\hline title $(0.11 \%)$ & Title of the document \\
\hline txt & Document text (see text statistics in Table 2) \\
\hline txt_nwords & Number of words \\
\hline txt_nsentences & Number of sentences \\
\hline txt_nparagraphs & Number of paragraphs \\
\hline topic_k100 & The topic ID with highest gamma value within k100 LDA ( $N=100$ unique, e.g., k100_24) \\
\hline topic_k200 & The topic ID with highest gamma value within k200 LDA ( $N=200$ unique, e.g., k200_75) \\
\hline topic_k300 & The topic ID with highest gamma value within k300 LDA ( $N=300$ unique, e.g., k300_192) \\
\hline mention_conspiracy & Occurrences count for the word "conspir" in text, see "Mentioning conspiracy" " section \\
\hline conspiracy_representative & Logical. TRUE $(N=4227)$ if the conspiracy document is representative \\
\hline cosine_similarity & Cosine similarity values for conspiracy documents (values $>$ mean +1 SD are considered representative) \\
\hline FB_shares $(0.01 \%)$ & URL's Facebook shares \\
\hline FB_comments $(0.01 \%)$ & URL's Facebook comments \\
\hline FB_reactions $(0.01 \%)$ & URL's Facebook reactions \\
\hline
\end{tabular}

Note. Percentages of empty/missing values are calculated on the list of documents $(N=96,743)$

Table 7 LOCO's website metadata variables description

\begin{tabular}{|c|c|}
\hline $\begin{array}{l}\text { Variable name }(\% \text { empty/missing values, if } \\
\text { any) }\end{array}$ & Variable description \\
\hline Website & Website name $(N=150)$ \\
\hline URL & URL associated with the website domain \\
\hline n_webpages & Overall number of webpages in website obtained by Google search (see "Website metrics" section) \\
\hline MBFC_political_orientation (69\%) & Political orientation. Left $(N=4)$, left_center $(N=19)$, least_biased $(N=15)$, right_center $(N=4)$, right $(N=5)$ \\
\hline MBFC_factual_reporting (21\%) & $\begin{array}{l}\text { Factual reporting. Very_low }(N=10) \text {, low }(N=43) \text {, mixed }(N=16) \text {, mostly_factual }(N=4) \text {, high }(N=35) \text {, very_high }(N= \\
\text { 11) }\end{array}$ \\
\hline MBFC_conspiracy & Logical. If TRUE $(N=58)$, website is conspiracy \\
\hline MBFC_pseudoscience (62\%) & For conspiracy websites only. Zero $(N=1)$, mild $(N=2)$, moderate $(N=9)$, strong $(N=16)$, quackery $(N=29)$ \\
\hline MBFC_proscience & Logical. TRUE $(N=16)$ if website is labeled as pro-science \\
\hline SW_total_visits (20\%) & Total visits, desktop and mobile web aggregated \\
\hline SW_global_rank (22\%) & Traffic rank of website, as compared to all other websites in the world \\
\hline SW_Category (20\%) & Website category (e.g., news_and_media, $N=60$; health, $N=16$, science_and_education, $N=13$ ) \\
\hline SW_traffic_direct (20\%) & Percentage of direct desktop incoming traffic (from typing the URL in a browser) \\
\hline SW_traffic_search (20\%) & Percentage of search desktop incoming traffic (from a search engine) \\
\hline SW_traffic_social (20\%) & Percentage of direct desktop incoming traffic (from a URL on social media) \\
\hline FB_shares_homepage & Facebook shares of homepage (see discussion in SM9) \\
\hline FB_shares_estimated & Estimated overall Facebook shares given total number of website's webpages (see "Facebook shares" section) \\
\hline
\end{tabular}

Note. Percentages of empty/missing values are calculated on the list of websites $(N=150)$ 
(2020-03-11, k300 200 and k300 288); and other significant societal events such as the 9/11 terroristic attack (2001-09-11, k300_72), the Sandy Hook school shooting (2012-12-14, k300 290), and Pizzagate (2016-11-01, when it went viral, k300_192). In Fig. 2, for all documents in LOCO provided with upload/creation data, topic patterns (i.e., gamma values on the $Y$ axis) are shown within a time span of 25 years, from 1995 to 2020 (first three rows) and for 2020 (fourth row for coronavirus-related topics) from January to July, when LOCO's data collection ended.

\section{Lexical features}

\section{Overlap with Reddit users' language}

Ideally, a corpus must be representative and replicable, meaning that the sampled data should represent the full range of variability of the population from which the sample is drawn. If our corpus successfully represents CTs, then its content should mirror the content of comments and threads posted by conspiracy believers on social media. To this aim, we compared the lexical features extracted from LOCO's documents (LOCO_LFs.json) with those extracted from comments on Reddit by Klein et al. (2019). Although user discussions on conspiracy forums are not conspiracy per se, we expect a certain overlap in language features with LOCO documents. This is because, while forums do not offer adequate space to fully develop argumentative discourses, a conspiracy believer can nevertheless express a conspiratorial worldview through language use (e.g., deception: "They are hiding the cure from us for their own profit!!'), even in discussion not related to conspiracy. In fact, Klein et al. (2019) compared language features of a group of users who posted in the r/conspiracy subreddit with those from a carefully matched control group of users who never posted in r/conspiracy. Although we do not know to what extent users who posted in the r/conspiracy subreddit endorse CTs, Klein and colleagues found language differences associated with a conspiratorial mindset (e.g., power, deception, dominance) that sees hidden powerful and malevolent enemies at work.

We proceeded with replicating the method of Klein et al. (2019) on LOCO by comparing our two subcorpora and explored whether the same patterns emerged. Similar to their work, we used the lexical features derived from Empath and tested differences between conspiracy and mainstream documents on the 194 Empath categories. Then, we used Welch's $t$-test and computed Cohen's $d$ for each test on the variables that yielded a significant difference at $p<.00026$ (Bonferroni correction for 194 tests). Note that here we are not testing any particular hypothesis, but provide this as exploratory analysis to guide future research. Results are shown in Fig. 3. On the top (A), only variables that produced an effect size of $d>.20$ are displayed, arranged in decreasing order. On the bottom
(B), each variable was scaled to $z$ values, and mean values are shown for different website categories: conspiracy_representative $(N=4,227)$, other conspiracy $(N$ $=19,710)$, biased LR (aggregating documents biased towards either the left or right, $N=31,928)$, least-biased $(N$ $=14,180)$, and pro-science $(N=11,440){ }^{17}$

Lexical differences between LOCO conspiracy and mainstream documents overlap with those between Reddit groups found by Klein and collaborators (Fig. 3a). Among the lexical categories characterizing conspiracy language (i.e., positive values in Fig. 3a), half of them emerged as overlapping between the two datasets. In LOCO, other lexical categories were higher in conspiracy (vs. mainstream), such as divine and worship that correlate with religion $(r=.92, r=.95$, respectively, in our dataset) found in Klein, and kill and hate that correlate with death $(r=.72, r=.44)$ and negative_emotion $(r=.71 ; r=.76)$ found in Klein but not in LOCO. It is also worth noting that representative conspiracy documents, on average, display an exaggeration of the "average" conspiratorial language as exemplified from the means further departing from zero (this will be further explored in "Properties of representative conspiracy documents" section).

\section{Effect of mentioning conspiracy}

We explored the possibility that mentioning conspiracy in the text would increase conspiratorial language (see "Mentioning "conspiracy" section). To this aim, we run multiple $t$-tests using as dependent variables the 31 lexical categories that yielded an effect size $d>.20$ from the previous section (see "Overlap with Reddit users"" section). In doing so, we used two different LOCO subcorpora: one (raw) which is based on the whole mainstream data $\left(N_{\text {mainstream }}=72,806\right)$ and one (cleaned) from which we removed all mainstream documents containing at least one instance of the word "conspir*" (Final $\left.N_{\text {mainstream }}=67,775\right)$. Note that we removed mentions of conspiracy only in the mainstream documents because we aimed to test the difference between the subcorpora removing potential conspiratorial language from mainstream documents, so as to obtain a mainstream subcorpus cleaned of conspiratorial language. We reasoned that conspiracy documents deliver conspiracy language even without mentioning the word "conspir*." From each test, we extracted the effect size (Cohen's $d$ ) and then compared the changes in $d$, with a paired $t$-test, from the raw to the cleaned dataset. Results show an overall increase in the effect sizes, $t_{(30)}=5.08, p<.001$, suggesting that cleaning the mainstream subcorpus from

\footnotetext{
${ }^{17}$ Note that the sum of pro-science, least-biased, and biased documents is 57,575 (and not 72,806, the total of mainstream documents). This is because not all websites are rated by MBFC, and therefore documents from these websites do not compare in plot $\mathrm{B}$.
} 
A)

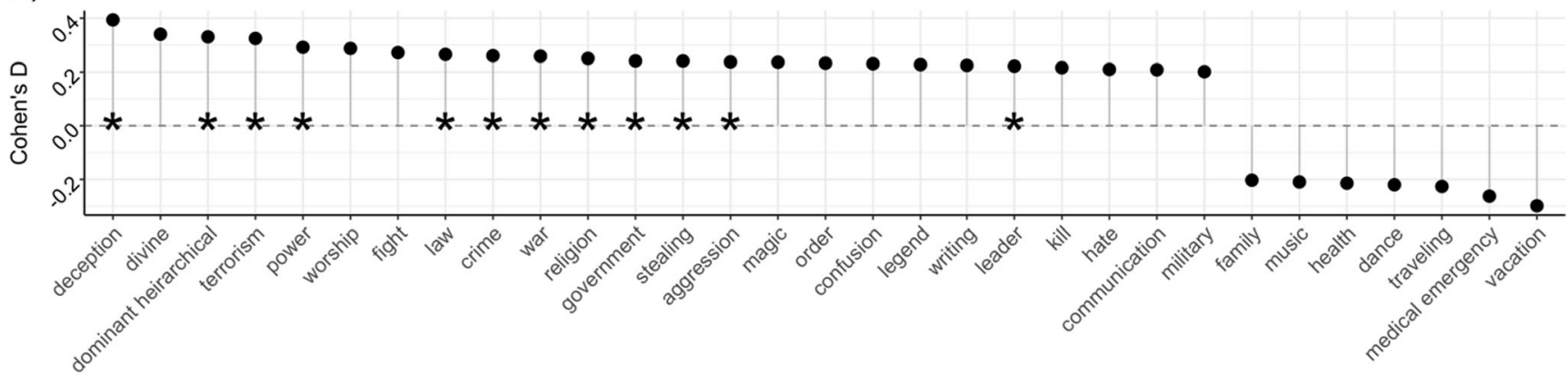

B)

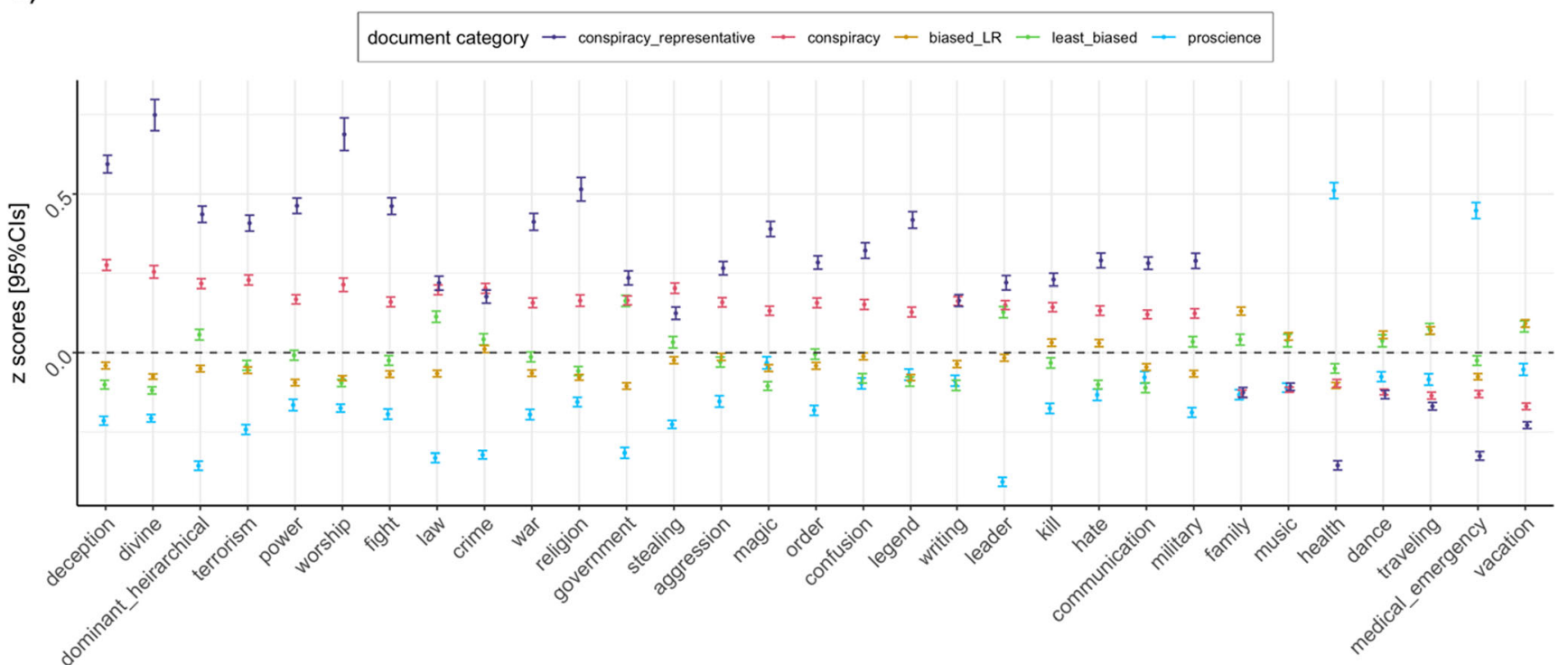

Empath categories

Fig. 3 Differences in lexical features between conspiracy and mainstream documents a Effect sizes that yielded a Cohen's $d>.20$ from $t$-tests between conspiracy and mainstream documents on Empath lexical categories. Positive effect sizes indicate that the category value is higher

documents that mention conspiracies amplifies differences in language features between the two subcorpora (see SM10 for details).

We finally explored whether mentioning conspiracy had an effect on lexical features. To this aim, we extracted the top four Empath categories that in the previous analysis (see paragraph above) had yielded the largest changes in effect size, namely crime, terrorism, deception, and stealing, and tested the correlation (on log-transformed variables, but see SM10 for non-log-transformed results) between the number of mentions of conspiracy and lexical variables. The results showed a positive relationship: crime: $r=.31$; terrorism: $r=.33$; deception: $r=.21$; and stealing: $r=.13$. Overall, these tests show that mentioning conspiracy, even in mainstream documents, affects language features. Therefore, we suggest that researchers carefully evaluate whether or not to include mainstream documents containing the word "conspir*" in their analyses. in conspiracy documents. A star indicates that the category emerged as having $d>.20$ also in Klein et al. (2019). b Comparison of means [and $95 \%$ CIs] for the same set of variables (scaled to $z$ values) across different document categories

\section{Properties of representative conspiracy documents}

We explored to what extent the representative set of conspiracy documents $(N=4,227)$ differs from the other conspiracy documents $(N=19,710)$ in terms of lexical features. To this aim, after subsetting LOCO to only conspiracy documents, we run a series of linear mixed-effects models using the lme 4 (Bates et al., 2015) and the lmerTest (Kuznetsova et al., 2017) R packages. In each model, we specified as dependent variables the LIWC $(N=93)$ and Empath $(N=194)$ categories, and as fixed effects the dichotomous representativeness predictor. As random intercept, we specified both the websites from which documents were extracted and the topic label with the highest gamma value for $k=100$ because, being less specific, it provides a more inclusive clustering that aggregates similar topics. In other words, while for $k=300$ we would have had several LDA topics revolving around a theme, with a lower topic resolution, topics are more general 
(we replicated the same analyses with $k=200$ topics, and results are not visibly different, see SM11). Before entering the model, the dependent variables were scaled to $z$ values. Standardized $\beta$ estimates are displayed in Fig. 4 for only the dependent variables that were significant at $p<.00017$ (Bonferroni correction for 287 tests) by the dichotomous predictor. Positive estimates indicate that the category is higher in the representative subset of conspiracy documents.

The representative conspiracy subset is generally more emotionally charged than the other documents, as displayed by the higher value for the category related to affective processes (LIWC category affect), and more specifically to negative emotions (LIWC categories anger, swear, negemo). Representative conspiracy documents, as compared with the non-representative conspiracy documents, display a prototypical language of conspiracy focused on power, dominance, and aggression (Empath categories deception, dominant hierarchical, kill, hate, order, power, aggression, and rage).

As for the rhetorical style used by the representative subset, we observe higher values for certainty (category certain), and interrogative (category interrog) language, along with higher use of question and exclamation marks (categories Exclam, QMark). This is in line with the observation that the rhetorical style of conspiracy narratives is built upon refutational strategies based on questioning the dubious version of the official story while highlighting the lack of answers from official sources (Oswald, 2016).

In line with research on social motives underlying belief in CTs (Douglas et al., 2019), the higher use of we and they, along with affiliative (LIWC category affiliation) and social (category social) language, suggests a process of social identification of the ingroup (we) by exclusion from the outgroup (they).

Overall, as already seen in Fig. 3 and in the work of Klein et al. (2019), the representative conspiracy documents seem to be an exaggerated version of an average conspiracy document, characterized by language of power, action, and dominance. They are at the same time less likely to display nonconspiratorial language as exemplified by lower values for categories such as tourism, vacation, urban, and morning. Interestingly, these patterns overlap with those found on Twitter, in which lexical differences between conspiracy and science influencers were identified in the use of negative emotion (e.g., anger) and a focus on topics such as death, religion, and power (Fong et al., 2021).

If the representative documents are rhetorically appealing and emotionally loaded, then we can expect that they will spread more successfully than the other, less representative documents. This reasoning is also in line with the fact that emotional content is a successful feature of narrative stickiness and transmission (Franks et al., 2013; Heath et al., 2001). Therefore, we tested whether the representative subset of conspiracy documents spread more than non-representative conspiracy documents. To this aim, we fit a linear mixed-effects model predicting Facebook shares (log-transformed). We set conspiracy representativeness as predictor along with website total visits as covariate while specifying a random intercept for websites. Results showed that the subset of representative
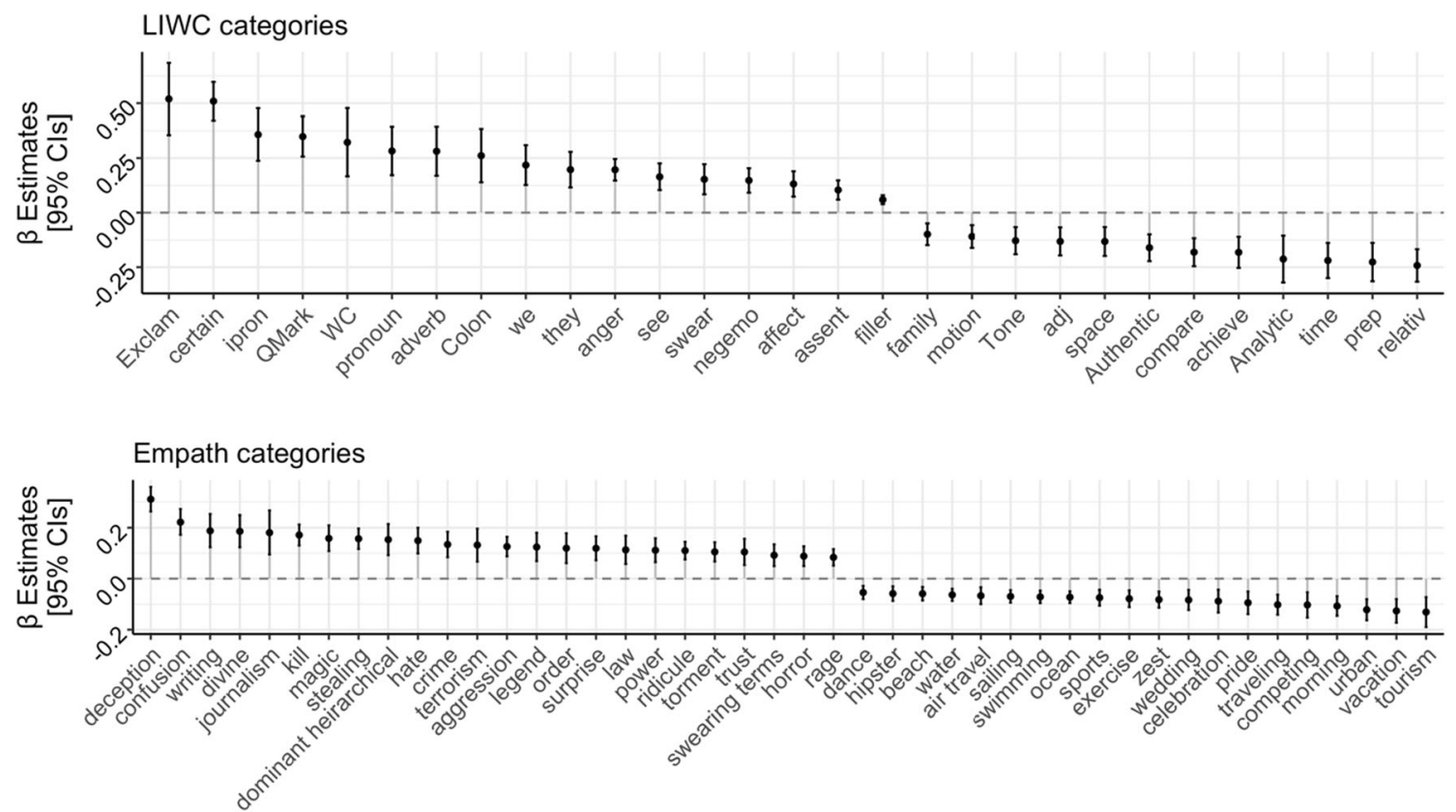

Fig. 4 Differences in lexical features between high- and lowrepresentative conspiracy documents. Positive $\beta$ estimates indicate that the category is higher among conspiracy documents that are more representative of the conspiracy corpus as measured by their document cosine similarity with other conspiracy documents in the corpus 
conspiracy documents was more shared on Facebook compared to the other conspiracy documents, $\beta=0.121, S E=$ $0.017, t=7.075, p<.001$.

\section{Website incoming traffic}

Besides the texts themselves and the documents and their metadata, LOCO is also provided with higher-level metadata, namely information about its constituent websites. Such a set of variables (contained in the file website_metadata.json) might be useful for testing hypotheses at the website level. For example, here, we describe the behavior of conspiracy and non-conspiracy communities from websites traffic information.

Analysis of online social media shows that users tend to aggregate in echo chambers that are homogeneous clusters of communities of interest (Bessi, Coletto, et al., 2015a; Brugnoli et al., 2019; Del Vicario, Bessi, et al., 2016a). Such clustering is reinforced in online and offline social networks (Del Vicario et al., 2017) whereby a like-minded trusted node in the social network (a friend or a page followed on Facebook) shares content that adheres to a system of beliefs. Moreover, within online social networks, users access information through a narrower spectrum of sources compared to web searches (Nikolov et al., 2015), meaning that being embedded within a social bubble reduces exposure to different viewpoints. When users of conspiracy Facebook pages are exposed to debunking information, they increase traffic towards conspiracy-like content (Zollo et al., 2017). This behavior suggests a confirmation bias: people avoid cognitive dissonance while searching for reinforcement (Brugnoli et al., 2019; Hills, 2019).

Website incoming traffic provides similar information about user behavior. For example, direct traffic may indicate a certain level of loyalty or at least that the user knows the website or has learned about it through their social contacts (Pauwels et al., 2016). When a website is reached from a search engine, the website is not necessarily known to the user. Put differently, how people arrive at a website may indirectly reveal information about their prior knowledge, beliefs, and social community. If echo chambers provide links to belief confirming content, then a confirmation bias theory of conspiratorial thinking would predict that users of conspiratorial websites are more likely to arrive there via a bookmarked URL or through online social networks than through impartial search engines.

To explore this possibility, we analyze user behavior through website incoming traffic (see "Website metrics" section). Because of a link between confirmation bias and belief in CTs (Del Vicario et al., 2017; Del Vicario, Bessi, et al., 2016a; Marchlewska et al., 2018; Meppelink et al., 2019; Zollo et al., 2017), we expect that conspiracy websites display higher levels of direct traffic and lower levels of search traffic.
Conspiracy ideas spread within homogeneous social media communities of like-minded believers who share conspiracy narratives; thus we also expect that traffic from social media (i.e., incoming traffic from a social media link) is higher in conspiracy compared to non-conspiracy websites. Moreover, because of known links between partisanship polarization and echo chambers (Stroud, 2010), confirmation bias (Westen et al., 2006), and belief in CTs (van Prooijen et al., 2015) we explored whether politically polarized websites (on both left and right sides of the spectrum) show patterns comparable to those of conspiracy websites compared to least biased websites.

We selected the websites (for which traffic data were collected) labeled as conspiracy $(N=28)$, least biased $(N=15)$, and pro-science $(N=16)$, and aggregated the websites leaning on either the left or right of the political spectrum, labeling them "biased_LR" $(N=32)$. Analysis of variance and post hoc comparisons using Tukey's honestly significant difference (HSD) test were used to test differences in traffic type between website categories. Direct traffic was highest for conspiracy $(M=57.55, S D=21.57)$ and lowest for pro-science $(M=13.35, S D=12.12), F_{(3,87)}=23.41, p<.001$. All post hoc differences among the four categories were significant at $p<.01$ except differences between least biased and biased websites $(p=.92)$ and between pro-science and least biased websites $(p=.09)$. As for traffic from search engines, the highest rate was on pro-science websites $(M=70.80, S D=$ $14.80)$ and the lower on conspiracy ones $(M=13.82, S D=$ $10.44), F_{(3,87)}=70.46, p<.001$. All differences were significant $(p s<.001)$ except those between least biased and biased websites $(\mathrm{p}=.76)$. Incoming traffic from social media sites was higher in conspiracy $(M=18.40, S D=19.28)$ than in pro-science $(M=5.44, S D=4.76), F_{(3,87)}=4.93, p<.01$; all other differences were nonsignificant. Results are shown in Fig. 5.

These results suggest that CT websites are predominantly reached by the users typing the URL on their browser (or by recalling the URL from bookmarks) or following a link posted on social media. On the contrary, pro-science websites are mostly accessed from web searches. Differences in access routes between biased and least-biased websites were not significant. This indicates that though users of conspiracy websites are most similar to users of biased websites, they are nonetheless in a category of their own.

\section{Discussion and conclusions}

LOCO is a multilevel, richly annotated, topic-matched corpus of CTs composed of nearly 100,000 documents, with a total of 88 million words. This represents a rich source of data for better understanding the content and spread of CTs. LOCO is also freely available (https://osf.io/snpcg). Being for the 


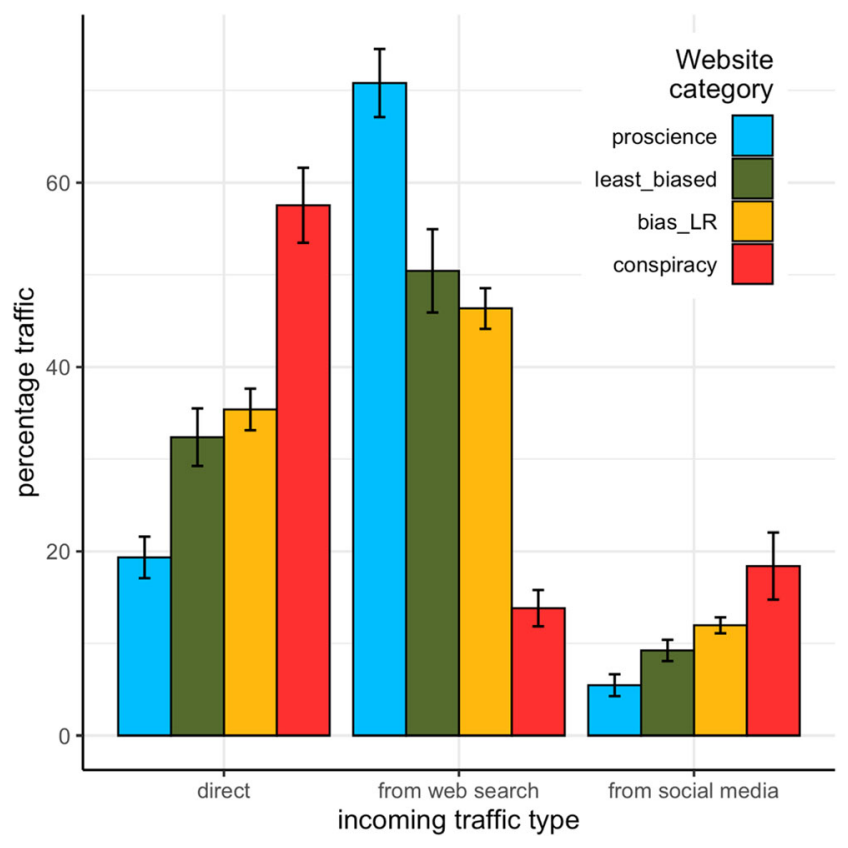

Fig. 5 Types of incoming traffic by website category. Average of websites' percentages of incoming traffic (direct, from web search, and from social media) by website categories. Error bars represent the standard error of the mean

most part composed of texts with additional metadata and lexical features, LOCO is conceptualized as a turnkey resource from which researchers can test hypotheses, further extract features, and/or build classification and predictive models. To this end, while building LOCO, we aimed at obtaining a large yet representative set of documents that also provided a set of metadata that could be used ad hoc to partition LOCO prior to analysis.

A large portion of the present paper has focused on thoroughly describing the methodology on which LOCO was built. As we have built LOCO upon previous works' strengths and weaknesses, we believe that a meticulous method description will also allow future research to benefit from LOCO's strengths and weaknesses, opening up possibilities for further data collection in the field of CT studies.

Our analysis of LOCO demonstrates its potential by making a number of contributions to the conspiracy research literature: (1) By mapping topics on document dates, we show that LOCO's documents track important social events. (2) We replicated the lexical analysis of previous work, finding an overlap between LOCO documents and comments on online social media. (3) We find that mainstream documents that mention conspiracy display conspiratorial language. (4) We have extracted and analyzed the language of prototypical conspiracy documents and find that these amplify features of conspiratorial language. (5) We find a pattern of website traffic indicating active online social media communities and the potential for confirmation bias via direct traffic. And (6) we find that conspiracy websites show statistically different patterns of web traffic than biased (politically left or right) websites, suggestive of a difference in their users. At the same time, we have provided suggestions on how to use LOCO to make new contributions.

Because we relied on a multitude of heterogeneous methods, we also believe that each of our corpus construction stages can benefit data collection for text analysis research in general. While we built LOCO on a specific narrative genre, namely CTs, the same methodology, or part of it, can be employed for other purposes. For example, researchers may be interested in comparing a list of websites against another one, or comparing webpages returned by specific sets of seeds, or, as we have done, do both at the same time by crossing lists of websites and seeds. We have also shown that it is possible to rely on several tools to enrich a web-based set of text with meta-data, such as political biases and fact accuracy (from MBFC), measures of spread (from SC), and popularity and traffic (from SW). Other freely available tools we have employed are available for text extraction (Goose) and analyses such as Empath, TAACO, as well as the quanteda and the topicmodels packages.

Because we also provided the URLs associated with each document, it is potentially possible to extract HTML data in order to analyze web markup features as previous work has done on fake news (Castelo et al., 2019). Moreover, different sets of psycholinguistic measures can be extracted from LOCO's texts, such as word norms for valence, arousal, and dominance (Warriner et al., 2013), imageability (Cortese \& Fugett, 2004), frequency (Brysbaert \& New, 2009), concreteness (Brysbaert et al., 2014), and age of acquisition (Kuperman et al., 2012).

In conclusion, LOCO is a rich source that helps to better understand the content of CTs. Here, we have explored how $\mathrm{CT}$ users behave online and which language features are associated with the spread of documents over social media, and we sketched a preliminary overview of the lexical fingerprint of the (prototypical) conspiratorial language. Therefore, LOCO's contribution is multiple: while providing data mainly for lexical analysis and document spread, it can also help to reveal psychological processes. For the sake of global public interest, given the detrimental potential consequences associated with the endorsement of CTs, understanding how CTs spread is critical to ultimately limiting their negative consequences.

Supplementary Information The online version contains supplementary material available at https://doi.org/10.3758/s13428-021-01698-z.

Funding Open Access funding provided by Université de Neuchâtel.

\section{Declarations}

Financial Disclosure None of the authors have any financial relationships relevant to this article to disclose. 
Open Access This article is licensed under a Creative Commons Attribution 4.0 International License, which permits use, sharing, adaptation, distribution and reproduction in any medium or format, as long as you give appropriate credit to the original author(s) and the source, provide a link to the Creative Commons licence, and indicate if changes were made. The images or other third party material in this article are included in the article's Creative Commons licence, unless indicated otherwise in a credit line to the material. If material is not included in the article's Creative Commons licence and your intended use is not permitted by statutory regulation or exceeds the permitted use, you will need to obtain permission directly from the copyright holder. To view a copy of this licence, visit http://creativecommons.org/licenses/by/4.0/.

\section{References}

Allen, C., \& Murdock, J. (2020). LDA Topic Modeling: Contexts for the History \& Philosophy of Science. http://philsci-archive.pitt.edu/ 17261/

Aston, G., \& Burnard, L. (1998). The BNC Handbook: Exploring the British National Corpus with SARA. In: English Language and Linguistics. Edinburgh University Press.

Aupers, S. (2012). 'Trust no one': Modernization, paranoia and conspiracy culture. European Journal of Communication, 27(1), 22-34. https://doi.org/10.1177/0267323111433566

AVAAZ. (2020). Facebook's Algorithm: A Major Threat to Public Health. https://secure.avaaz.org/campaign/en/facebook_threat health/

Baly, R., Karadzhov, G., Alexandrov, D., Glass, J., \& Nakov, P. (2018). Predicting Factuality of Reporting and Bias of News Media Sources. Proceedings of the 2018 Conference on Empirical Methods in Natural Language Processing, 3528-3539. https://doi.org/10. 18653/v1/D18-1389

Bangerter, A., Wagner-Egger, P., \& Delouvée, S. (2020). The Spread of Conspiracy Theories. In M. Butter \& P. Knight (Eds.), Routledge Handbook of Conspiracy Theories (pp. 2016-2218). Routledge.

Barkun, M. (2017). President Trump and the "Fringe". Terrorism and Political Violence, 29(3), 437-443. https://doi.org/10.1080/ 09546553.2017.1313649

Baroni, M., Bernardini, S., Ferraresi, A., \& Zanchetta, E. (2009). The WaCky wide web: a collection of very large linguistically processed web-crawled corpora. Language Resources and Evaluation, 43(3), 209-226. https://doi.org/10.1007/s10579-009-9081-4

Barron, A. T. J., Huang, J., Spang, R. L., \& DeDeo, S. (2018). Individuals, institutions, and innovation in the debates of the French Revolution. Proceedings of the National Academy of Sciences, 115(18), 4607-4612. https://doi.org/10.1073/pnas. 1717729115

Bates, D., Mächler, M., Bolker, B., \& Walker, S. (2015). Fitting linear mixed-effects models using lme4. Journal of Statistical Software, 67(1), 1-48. https://doi.org/10.18637/jss.v067.i01

Bessi, A. (2016). Personality traits and echo chambers on facebook. Computers in Human Behavior, 65, 319-324. https://doi.org/10. 1016/j.chb.2016.08.016

Bessi, A., Scala, A., Rossi, L., Zhang, Q., \& Quattrociocchi, W. (2014). The economy of attention in the age of (mis)information. Journal of Trust Management, 1(1), 12. https://doi.org/10.1186/s40493-0140012-y

Bessi, A., Coletto, M., Davidescu, G. A., Scala, A., Caldarelli, G., \& Quattrociocchi, W. (2015a). Science vs Conspiracy: Collective Narratives in the Age of Misinformation. PLOS ONE, 10(2), e0118093. https://doi.org/10.1371/journal.pone.0118093

Bessi, A., Zollo, F., Del Vicario, M., Scala, A., Caldarelli, G., \& Quattrociocchi, W. (2015b). Trend of Narratives in the Age of
Misinformation. PLOS ONE, 10(8), e0134641. https://doi.org/10. 1371/journal.pone.0134641

Betsch, C., Ulshöfer, C., Renkewitz, F., \& Betsch, T. (2011). The Influence of Narrative v. Statistical Information on Perceiving Vaccination Risks. Medical Decision Making, 31(5), 742-753. https://doi.org/10.1177/0272989X11400419

Biddlestone, M., Green, R., \& Douglas, K. M. (2020). Cultural orientation, power, belief in conspiracy theories, and intentions to reduce the spread of COVID-19. British Journal of Social Psychology, 59(3), 663-673. https://doi.org/10.1111/bjso.12397

Bird, S., Klein, E., \& Loper, E. (2009). Natural language processing with Python: analyzing text with the natural language toolkit. "O'Reilly Media, Inc."

Blei, D. M., Ng, A. Y., \& Jordan, M. I. (2003). Latent Dirichlet allocation. Journal of Machine Learning Research, 3, 993-1022.

Bogart, L. M., Wagner, G., Galvan, F. H., \& Banks, D. (2010). Conspiracy Beliefs About HIV Are Related to Antiretroviral Treatment Nonadherence Among African American Men With HIV. JAIDS Journal of Acquired Immune Deficiency Syndromes, 53(5), 648-655. https://doi.org/10.1097/QAI.0b013e3181c57dbc

Brugnoli, E., Cinelli, M., Quattrociocchi, W., \& Scala, A. (2019). Recursive patterns in online echo chambers. Scientific Reports, 9(1), 20118. https://doi.org/10.1038/s41598-019-56191-7

Brysbaert, M., \& New, B. (2009). Moving beyond Kučera and Francis: A critical evaluation of current word frequency norms and the introduction of a new and improved word frequency measure for American English. Behavior Research Methods, 41(4), 977-990. https://doi.org/10.3758/BRM.41.4.977

Brysbaert, M., Warriner, A. B., \& Kuperman, V. (2014). Concreteness ratings for 40 thousand generally known English word lemmas. Behavior Research Methods, 46(3), 904-911. https://doi.org/10. 3758/s13428-013-0403-5

Butter, M., \& Knight, P. (2020). Routledge Handbook of Conspiracy Theories (M. Butter \& P. Knight (eds.)). Routledge.

Castelo, S., Santos, A., Almeida, T., Pham, K., Freire, J., Elghafari, A., \& Nakamura, E. (2019). A topic-agnostic approach for identifying fake news pages. The Web Conference 2019 - Companion of the World Wide Web Conference, WWW 2019. https://doi.org/10.1145/ 3308560.3316739

Cinelli, M., De Francisci Morales, G., Galeazzi, A., Quattrociocchi, W., \& Starnini, M. (2021). The echo chamber effect on social media. Proceedings of the National Academy of Sciences, 118(9), e2023301118. https://doi.org/10.1073/pnas.2023301118

Clarke, S. (2007). Conspiracy Theories and the Internet: Controlled Demolition and Arrested Development. Episteme, 4(2), 167-180. https://doi.org/10.3366/epi.2007.4.2.167

Cortese, M. J., \& Fugett, A. (2004). Imageability ratings for 3,000 monosyllabic words. Behavior Research Methods, Instruments, \& Computers, 36(3), 384-387. https://doi.org/10.3758/BF03195585

Crossley, S. A., Kyle, K., \& McNamara, D. S. (2016). The tool for the automatic analysis of text cohesion (TAACO): Automatic assessment of local, global, and text cohesion. Behavior Research Methods, 48(4), 1227-1237. https://doi.org/10.3758/s13428-0150651-7

Crossley, S. A., Kyle, K., \& Dascalu, M. (2019). The Tool for the Automatic Analysis of Cohesion 2.0: Integrating semantic similarity and text overlap. Behavior Research Methods, 51(1), 14-27. https:// doi.org/10.3758/s13428-018-1142-4

de Vries, E., Schoonvelde, M., \& Schumacher, G. (2018). No Longer Lost in Translation: Evidence that Google Translate Works for Comparative Bag-of-Words Text Applications. Political Analysis, 26(4), 417-430. https://doi.org/10.1017/pan.2018.26

Del Vicario, M., Bessi, A., Zollo, F., Petroni, F., Scala, A., Caldarelli, G., Stanley, H. E., \& Quattrociocchi, W. (2016a). The spreading of misinformation online. Proceedings of the National Academy of 
Sciences, 113(3), 554-559. https://doi.org/10.1073/pnas. 1517441113

Del Vicario, M., Vivaldo, G., Bessi, A., Zollo, F., Scala, A., Caldarelli, G., \& Quattrociocchi, W. (2016b). Echo Chambers: Emotional Contagion and Group Polarization on Facebook. Scientific Reports, 6(1), 37825. https://doi.org/10.1038/srep37825

Del Vicario, M., Scala, A., Caldarelli, G., Stanley, H. E., \& Quattrociocchi, W. (2017). Modeling confirmation bias and polarization. Scientific Reports, 7(1), 40391. https://doi.org/10.1038/ srep40391

Douglas, K. M., \& Sutton, R. M. (2011). Does it take one to know one? Endorsement of conspiracy theories is influenced by personal willingness to conspire. British Journal of Social Psychology, 50(3), 544-552.

Douglas, K. M., \& Sutton, R. M. (2018). Why conspiracy theories matter: A social psychological analysis. European Review of Social Psychology, 29(1), 256-298. https://doi.org/10.1080/10463283. 2018.1537428

Douglas, K. M., Uscinski, J. E., Sutton, R. M., Cichocka, A., Nefes, T., Ang, C. S., \& Deravi, F. (2019). Understanding Conspiracy Theories. Political Psychology, 40(S1), 3-35. https://doi.org/10. 1111/pops. 12568

Eicher, V., \& Bangerter, A. (2015). Social representations of infectious diseases. In G. Sammut, E. Andreouli, G. Gaskell, \& J. Valsiner (Eds.), The Cambridge Handbook of Social Representations (pp. 385-396). Cambridge University Press. https://doi.org/10.1017/ CBO9781107323650.031

Einstein, K. L., \& Glick, D. M. (2015). Do I Think BLS Data are BS? The Consequences of Conspiracy Theories. Political Behavior, 37(3), 679-701. https://doi.org/10.1007/s11109-014-9287-z

Faasse, K., Chatman, C. J., \& Martin, L. R. (2016). A comparison of language use in pro- and anti-vaccination comments in response to a high profile Facebook post,. Vaccine, 34(47), 5808-5814. https:// doi.org/10.1016/j.vaccine.2016.09.029

Fast, E., Chen, B., \& Bernstein, M. S. (2016). Empath. Proceedings of the 2016 CHI Conference on Human Factors in Computing Systems, 4647-4657. https://doi.org/10.1145/2858036.2858535

Fong, A., Roozenbeek, J., Goldwert, D., Rathje, S., \& van der Linden, S. (2021). The language of conspiracy: A psychological analysis of speech used by conspiracy theorists and their followers on Twitter. Group Processes \& Intergroup Relations, 24(4), 606-623. https:// doi.org/10.1177/1368430220987596

Franks, B., Bangerter, A., \& Bauer, M. W. (2013). Conspiracy theories as quasi-religious mentality: an integrated account from cognitive science, social representations theory, and frame theory. Frontiers in Psychology, 4, 1-12. https://doi.org/10.3389/fpsyg.2013.00424

Franks, B., Bangerter, A., Bauer, M. W., Hall, M., \& Noort, M. C. (2017). Beyond "Monologicality"? Exploring Conspiracist Worldviews. Frontiers in Psychology, 8. https://doi.org/10.3389/fpsyg.2017. 00861

Fry, E. (2000). 1000 instant words: the most common words for teaching reading, writing and spelling. Teacher Created Resources.

Fu, L. Y., Zook, K., Spoehr-Labutta, Z., Hu, P., \& Joseph, J. G. (2016). Search Engine Ranking, Quality, and Content of Web Pages That Are Critical Versus Noncritical of Human Papillomavirus Vaccine. Journal of Adolescent Health, 58(1), 33-39. https://doi.org/10.1016/ j.jadohealth.2015.09.016

Golec de Zavala, A., \& Cichocka, A. (2012). Collective narcissism and anti-Semitism in Poland. Group Processes \& Intergroup Relations, 15(2), 213-229. https://doi.org/10.1177/1368430211420891

Grün, B., \& Hornik, K. (2011). Topicmodels: An R package for fitting topic models. Journal of Statistical Software, 40(13), 1-30. https:// doi.org/10.18637/jss.v040.i13

Guerini, M., Giampiccolo, D., Moretti, G., Sprugnoli, R., \& Strapparava, C. (2013). The New Release of CORPS: A Corpus of Political Speeches Annotated with Audience Reactions. In: Lecture Notes in Computer Science (including subseries Lecture Notes in Artificial Intelligence and Lecture Notes in Bioinformatics) (pp. 86-98). https://doi.org/10.1007/978-3-642-41545-6_8

Heath, C., Bell, C., \& Sternberg, E. (2001). Emotional selection in memes: The case of urban legends. Journal of Personality and Social Psychology, 81(6), 1028-1041. https://doi.org/10.1037/ 0022-3514.81.6.1028

Hills, T. T. (2019). The Dark Side of Information Proliferation. Perspectives on Psychological Science, 14(3), 323-330. https:// doi.org/10.1177/1745691618803647

Imhoff, R., Lamberty, P., \& Klein, O. (2018). Using Power as a Negative Cue: How Conspiracy Mentality Affects Epistemic Trust in Sources of Historical Knowledge. Personality and Social Psychology Bulletin, 44(9), 1364-1379. https://doi.org/10.1177/ 0146167218768779

Imhoff, R., Dieterle, L., \& Lamberty, P. (2021). Resolving the Puzzle of Conspiracy Worldview and Political Activism: Belief in Secret Plots Decreases Normative but Increases Nonnormative Political Engagement. Social Psychological and Personality Science, 12(1), 71-79. https://doi.org/10.1177/1948550619896491

Jensen, T. (2013). Democrats and Republicans differ on conspiracy theory beliefs. Public Policy Polling. http://www.publicpolicypolling. $\mathrm{com} /$ polls/democrats-and-republicans-differ-on-conspiracy-theorybeliefs

Jolley, D., \& Douglas, K. M. (2014a). The social consequences of conspiracism: Exposure to conspiracy theories decreases intentions to engage in politics and to reduce one's carbon footprint. British Journal of Psychology, 105(1), 35-56. https://doi.org/10.1111/bjop. 12018

Jolley, D., \& Douglas, K. M. (2014b). The Effects of Anti-Vaccine Conspiracy Theories on Vaccination Intentions. PLOS ONE, 9(2), e89177. https://doi.org/10.1371/journal.pone.0089177

Jolley, D., \& Paterson, J. L. (2020). Pylons ablaze: Examining the role of 5G COVID-19 conspiracy beliefs and support for violence. British Journal of Social Psychology, 59(3), 628-640. https://doi.org/10. 1111/bjso.12394

Jolley, D., Douglas, K. M., Leite, A. C., \& Schrader, T. (2019). Belief in conspiracy theories and intentions to engage in everyday crime. British Journal of Social Psychology, 58(3), 534-549. https://doi. org/10.1111/bjso.12311

Klein, C., Clutton, P., \& Polito, V. (2018). Topic Modeling Reveals Distinct Interests within an Online Conspiracy Forum. Frontiers in Psychology, 9. https://doi.org/10.3389/fpsyg.2018.00189

Klein, C., Clutton, P., \& Dunn, A. G. (2019). Pathways to conspiracy: The social and linguistic precursors of involvement in Reddit's conspiracy theory forum. PLOS ONE, 14(11), e0225098. https://doi. org/10.1371/journal.pone.0225098

Kuperman, V., Stadthagen-Gonzalez, H., \& Brysbaert, M. (2012). Ageof-acquisition ratings for 30,000 English words. Behavior Research Methods, 44(4), 978-990. https://doi.org/10.3758/s13428-0120210-4

Kuznetsova, A., Brockhoff, P. B., \& Christensen, R. H. B. (2017). lmerTest Package: Tests in Linear Mixed Effects Models. Journal of Statistical Software, 82(13). https://doi.org/10.18637/jss.v082.i13

Kwon, S., Cha, M., \& Jung, K. (2017). Rumor Detection over Varying Time Windows. PLOS ONE, 12(1), e0168344. https://doi.org/10. 1371/journal.pone.0168344

Kyle, K., Crossley, S. A., \& Kim, Y. J. (2015). Native language identification and writing proficiency. International Journal of Learner Corpus Research, 1(2), 187-209. https://doi.org/10.1075/ijlcr.1.2. $01 \mathrm{kyl}$

Lansdall-Welfare, T., Sudhahar, S., Thompson, J., Lewis, J., \& Cristianini, N. (2017). Content analysis of 150 years of British periodicals. Proceedings of the National Academy of Sciences, 114(4), E457-E465. https://doi.org/10.1073/pnas.1606380114 
Lantian, A., Muller, D., Nurra, C., Klein, O., Berjot, S., \& Pantazi, M. (2018). Stigmatized beliefs: Conspiracy theories, anticipated negative evaluation of the self, and fear of social exclusion. European Journal of Social Psychology, 48(7), 939-954. https://doi.org/10. 1002/ejsp.2498

Lazarus, J. V., Ratzan, S. C., Palayew, A., Gostin, L. O., Larson, H. J., Rabin, K., Kimball, S., \& El-Mohandes, A. (2020). A global survey of potential acceptance of a COVID-19 vaccine. Nature Medicine. https://doi.org/10.1038/s41591-020-1124-9

Li, Y., Engelthaler, T., Siew, C. S. Q., \& Hills, T. T. (2019). The Macroscope: A tool for examining the historical structure of language. Behavior Research Methods, 51(4), 1864-1877. https://doi. org/10.3758/s13428-018-1177-6

Li, Y., Hills, T., \& Hertwig, R. (2020). A brief history of risk. Cognition, 203, 104344. https://doi.org/10.1016/j.cognition.2020.104344

Marchlewska, M., Cichocka, A., \& Kossowska, M. (2018). Addicted to answers: Need for cognitive closure and the endorsement of conspiracy beliefs. European Journal of Social Psychology. https://doi. org/10.1002/ejsp.2308

Meppelink, C. S., Smit, E. G., Fransen, M. L., \& Diviani, N. (2019). "I was Right about Vaccination": Confirmation Bias and Health Literacy in Online Health Information Seeking. Journal of Health Communication, 24(2), 129-140. https://doi.org/10.1080/ 10810730.2019.1583701

Michel, J.-B., Shen, Y. K., Aiden, A. P., Veres, A., Gray, M. K., Pickett, J. P., Hoiberg, D., Clancy, D., Norvig, P., Orwant, J., Pinker, S., Nowak, M. A., \& Aiden, E. L. (2011). Quantitative Analysis of Culture Using Millions of Digitized Books. Science, 331(6014), 176-182. https://doi.org/10.1126/science.1199644

Mitra, T., Counts, S., \& Pennebaker, J. W. (2016). Understanding antivaccination attitudes in social media. Proceedings of the 10th International Conference on Web and Social Media, ICWSM 2016.

Nguyen, D., Liakata, M., DeDeo, S., Eisenstein, J., Mimno, D., Tromble, R., \& Winters, J. (2020). How We Do Things With Words: Analyzing Text as Social and Cultural Data. Frontiers in Artificial Intelligence, 3. https://doi.org/10.3389/frai.2020.00062

Nikolov, D., Oliveira, D. F. M., Flammini, A., \& Menczer, F. (2015). Measuring online social bubbles. PeerJ Computer Science, 1(12), e38. https://doi.org/10.7717/peerj-cs.38

Okuhara, T., Ishikawa, H., Okada, M., Kato, M., \& Kiuchi, T. (2017). Readability comparison of pro- and anti-HPV-vaccination online messages in Japan. Patient Education and Counseling, 100(10), 1859-1866. https://doi.org/10.1016/j.pec.2017.04.013

Oliver, J. E., \& Wood, T. J. (2014). Conspiracy Theories and the Paranoid Style(s) of Mass Opinion. American Journal of Political Science, 58(4), 952-966. https://doi.org/10.1111/ajps.12084

Ooms, J. (2019). curl: A Modern and Flexible Web Client for R. https:// cran.r-project.org/package $=$ curl

Oswald, S. (2016). Conspiracy and bias: argumentative features and persuasiveness of conspiracy theories. OSSA Conference Archive, 168, $1-16$.

Pauwels, K., Demirci, C., Yildirim, G., \& Srinivasan, S. (2016). The impact of brand familiarity on online and offline media synergy. International Journal of Research in Marketing, 33(4), 739-753. https://doi.org/10.1016/j.ijresmar.2015.12.008

Pennycook, G., \& Rand, D. G. (2019). Fighting misinformation on social media using crowdsourced judgments of news source quality. Proceedings of the National Academy of Sciences, 116(7), 25212526. https://doi.org/10.1073/pnas.1806781116

Perez, J. C., \& Montagnier, L. (2020). Covid-19, Sars And Bats Coronaviruses Genomes Peculiar Homologous RNA Sequences. International Journal of Research -GRANTHAALAYAH, 8(7), 217-263. https://doi.org/10.29121/granthaalayah.v8.i7.2020.678

R Core Team. (2019). R: A Language and Environment for Statistical Computing. https://www.r-project.org/
Raab, M. H., Auer, N., Ortlieb, S. A., \& Carbon, C.-C. (2013a). The Sarrazin effect: the presence of absurd statements in conspiracy theories makes canonical information less plausible. Frontiers in Psychology, 4, 1-8. https://doi.org/10.3389/fpsyg.2013.00453

Raab, M. H., Ortlieb, S. A., Auer, N., Guthmann, K., \& Carbon, C.-C. (2013b). Thirty shades of truth: conspiracy theories as stories of individuation, not of pathological delusion. Frontiers in Psychology, 4. https://doi.org/10.3389/fpsyg.2013.00406

Risius, M., Aydinguel, O., \& Haug, M. (2019). Towards an understanding of conspiracy echo chambers on Facebook. Proceedings of the 27th European Conference on Information Systems (ECIS). https:// aisel.aisnet.org/ecis2019_rip/36

Sak, G., Diviani, N., Allam, A., \& Schulz, P. J. (2015). Comparing the quality of pro- and anti-vaccination online information: a content analysis of vaccination-related webpages. BMC Public Health, 16(1), 38. https://doi.org/10.1186/s12889-016-2722-9

Salmon, D. A., Moulton, L. H., Omer, S. B., DeHart, M. P., Stokley, S., \& Halsey, N. A. (2005). Factors associated with refusal of childhood vaccines among parents of school-aged children: a case-control study. Archives of Pediatrics \& Adolescent Medicine, 159(5), 470-476. https://doi.org/10.1001/archpedi.159.5.470

Samory, M., \& Mitra, T. (2018a). Conspiracies online: User discussions in a conspiracy community following dramatic events. 12th International AAAI Conference on Web and Social Media, ICWSM 2018.

Samory, M., \& Mitra, T. (2018b). "The Government Spies Using Our Webcams:" The language of conspiracy theories in online discussions. Proceedings of the ACM on Human-Computer Interaction, 2(152). https://doi.org/10.1145/3274421

Smith, N., \& Graham, T. (2019). Mapping the anti-vaccination movement on Facebook. Information, Communication \& Society, 22(9), 1310-1327. https://doi.org/10.1080/1369118X.2017.1418406

Sternisko, A., Cichocka, A., \& Van Bavel, J. J. (2020). The dark side of social movements: social identity, non-conformity, and the lure of conspiracy theories. Current Opinion in Psychology, 35, 1-6. https://doi.org/10.1016/j.copsyc.2020.02.007

Stroud, N. J. (2010). Polarization and Partisan Selective Exposure. Journal of Communication, 60(3), 556-576. https://doi.org/10. 1111/j.1460-2466.2010.01497.x

Swami, V., Barron, D., Weis, L., \& Furnham, A. (2018). To Brexit or not to Brexit: The roles of Islamophobia, conspiracist beliefs, and integrated threat in voting intentions for the United Kingdom European Union membership referendum. British Journal of Psychology, 109(1), 156-179. https://doi.org/10.1111/bjop.12252

Tausczik, Y. R., \& Pennebaker, J. W. (2010). The psychological meaning of words: LIWC and computerized text analysis methods. Journal of Language and Social Psychology, 29(1), 24-54. https://doi.org/10. 1177/0261927X09351676

Uscinski, J. E., Parent, J. M., \& Torres, B. (2011). Conspiracy Theories Are for Losers. APSA 2011 Annual Meeting Paper. https://ssrn.com/ abstract $=1901755$

Uscinski, J. E., DeWitt, D., \& Atkinson, M. D. (2018). A Web of Conspiracy? Internet and Conspiracy Theory. In: Handbook of Conspiracy Theory and Contemporary Religion (pp. 106-130). BRILL. https://doi.org/10.1163/9789004382022 007

van der Linden, S. (2015). The conspiracy-effect: Exposure to conspiracy theories (about global warming) decreases pro-social behavior and science acceptance. Personality and Individual Differences, 87, 171-173. https://doi.org/10.1016/j.paid.2015.07.045

van Prooijen, J.-W., Krouwel, A. P. M., \& Pollet, T. V. (2015). Political Extremism Predicts Belief in Conspiracy Theories. Social Psychological and Personality Science, 6(5), 570-578. https://doi. org $/ 10.1177 / 1948550614567356$

von Luxburg, U., Williamson, R. C., \& Guyon, I. (2012). Clustering: Science or Art? In I. Guyon, G. Dror, V. Lemaire, G. Taylor, \& D. Silver (Eds.), Proceedings of ICML Workshop on Unsupervised and 
Transfer Learning (Vol. 27, pp. 65-79). PMLR. http://proceedings. mlr.press/v27/luxburg12a.html

Vosoughi, S., Roy, D., \& Aral, S. (2018). The spread of true and false news online. Science, 359(6380), 1146-1151. https://doi.org/10. 1126/science.aap9559

Wakefield, A., Murch, S., Anthony, A., Linnell, J., Casson, D., Malik, M., Berelowitz, M., Dhillon, A., Thomson, M., Harvey, P., Valentine, A., Davies, S., \& Walker-Smith, J. (1998). RETRACTED: Ileal-lymphoid-nodular hyperplasia, non-specific colitis, and pervasive developmental disorder in children. The Lancet, 351(9103), 637-641. https://doi.org/10.1016/S01406736(97)11096-0

Warriner, A. B., Kuperman, V., \& Brysbaert, M. (2013). Norms of valence, arousal, and dominance for 13,915 English lemmas. Behavior Research Methods, 45(4), 1191-1207. https://doi.org/10.3758/ s13428-012-0314-x

Westen, D., Blagov, P. S., Harenski, K., Kilts, C., \& Hamann, S. (2006). Neural Bases of Motivated Reasoning: An fMRI Study of Emotional Constraints on Partisan Political Judgment in the 2004 U.S. Presidential Election. Journal of Cognitive Neuroscience, 18(11), 1947-1958. https://doi.org/10.1162/jocn.2006.18.11.1947

Wood, M. J. (2018). Propagating and Debunking Conspiracy Theories on Twitter During the 2015-2016 Zika Virus Outbreak.
Cyberpsychology, Behavior, and Social Networking, 21(8), 485490. https://doi.org/10.1089/cyber.2017.0669

Wood, M. J., \& Douglas, K. M. (2013). What about building 7? A social psychological study of online discussion of 9/11 conspiracy theories. Frontiers in Psychology, 4, 1-9. https://doi.org/10.3389/fpsyg. 2013.00409

Wood, M. J., \& Douglas, K. M. (2015). Online communication as a window to conspiracist worldviews. Frontiers in Psychology, 6. https://doi.org/10.3389/fpsyg.2015.00836

Zannettou, S., Bradlyn, B., De Cristofaro, E., Kwak, H., Sirivianos, M., Stringini, G., \& Blackburn, J. (2018). What is Gab. Companion of the Web Conference 2018 on The Web Conference 2018 - WWW '18, 1007-1014. https://doi.org/10.1145/3184558.3191531

Zollo, F., Bessi, A., Del Vicario, M., Scala, A., Caldarelli, G., Shekhtman, L., Havlin, S., \& Quattrociocchi, W. (2017). Debunking in a world of tribes. PLOS ONE. https://doi.org/10.1371/journal.pone.0181821

Zubiaga, A., Liakata, M., Procter, R., Wong Sak Hoi, G., \& Tolmie, P. (2016). Analysing How People Orient to and Spread Rumours in Social Media by Looking at Conversational Threads. PLOS ONE, 11(3), e0150989. https://doi.org/10.1371/journal.pone.0150989

Publisher's note Springer Nature remains neutral with regard to jurisdictional claims in published maps and institutional affiliations. 